\title{
On long-time existence for the flow of static metrics with rotational symmetry
}

\author{
Liljana Gulcev, Todd A. Oliynyk and Eric Woolgar
}

\begin{abstract}
B List has proposed a geometric flow whose fixed points correspond to solutions of the static Einstein equations of general relativity. This flow is now known to be a certain Hamilton-DeTurck flow (the pullback of a Ricci flow by an evolving diffeomorphism) on $\mathbb{R} \times$ $M^{n}$. We study the $\mathrm{SO}(n)$ rotationally symmetric case of List's flow under conditions of asymptotic flatness. We are led to this problem from considerations related to Bartnik's quasi-local mass definition and, as well, as a special case of the coupled Ricci-harmonic map flow. The problem also occurs as a Ricci flow with broken $\mathrm{SO}(n+$ 1) symmetry, and has arisen in a numerical study of Ricci flow for black hole thermodynamics. When the initial data admits no minimal hypersphere, we find the flow is immortal when a single regularity condition holds for the scalar field of List's flow at the origin. This regularity condition can be shown to hold at least for $n=2$. Otherwise, near a singularity, the flow will admit rescalings which converge to an $\mathrm{SO}(n)$-symmetric ancient Ricci flow on $\mathbb{R}^{n}$.
\end{abstract}

\section{Introduction}

\subsection{List's flow}

Many of the most exciting recent developments in geometric analysis have arisen from the study of geometric flow equations. Among the most prominent examples, the Ricci flow has yielded a proof of the Poincaré and Thurston conjectures $[11,26,30,31]$ and the diffeomorphic $\frac{1}{4}$-pinched sphere theorem [6], while the inverse mean curvature flow has been used to prove the Riemannian Penrose conjecture [18, 19]. The latter has important consequences in physics, prompting the question of whether other geometric flow problems might also arise from physics.

In physics one is often led to consider a metric of Lorentzian signature. Many geometric flow equations in Riemannian geometry are secondorder parabolic (or at least quasi-parabolic), and therefore they can be studied with the powerful tools of the maximum principle and entropies. 
The problem is that this power is generally lost in passing to Lorentzian signature.

However, Riemannian metrics can arise in physics problems, as the case of the Riemannian Penrose conjecture shows. To see how this can occur, consider the class of stationary spacetimes in general relativity. These spacetimes have metrics that admit a timelike Killing vector field. Examples are the Gödel and exterior Kerr metrics [16], in which there are preferred observers who view the time evolution of spacetime as nothing more than constant rotation. Quotienting a stationary spacetime by the isometry generated by the timelike Killing vector field, one obtains a Riemannian metric on the base manifold, which is smooth if the spacetime has no closed timelike curves and if the Killing orbits are complete [15].

If the timelike Killing field is hypersurface-orthogonal, we arrive at the class of static spacetimes, among which are the exterior Schwarzschild and flat Minkowski spacetimes. For static spacetimes, the rotation vanishes and the spacetime metric splits as a warped product of a one-dimensional fibre over a Riemannian base manifold. In particular, a static spacetime metric on $\mathbb{R} \times M^{n}, n>2$, can be written as

$$
d s^{2}=g_{\mu \nu} d x^{\mu} d x^{\nu}=-e^{2 u} d x^{0} d x^{0}+e^{-\frac{2 u}{n-2}} g_{i j} d x^{i} d x^{j}, \quad \frac{\partial u}{\partial x^{0}}=0, \quad \frac{\partial g_{i j}}{\partial x^{0}}=0 .
$$

Here $g_{\mu \nu}$ is a metric on $\mathbb{R} \times M^{n}$, while $g_{i j}$ is a metric on $M^{n}$. Coordinates on $M^{n+1}$ are $\left(x^{\mu}\right)=\left(x^{0}, x^{i}\right)$, so Greek indices run over one more value than Roman ones.

The vacuum Einstein equation is the condition that the metric (1.1) has vanishing Ricci curvature. If we apply the vacuum Einstein equation to the metric $g_{\mu \nu}$ in (1.1), we obtain the equations

$$
\begin{aligned}
& R_{i j}-\left(\frac{n-1}{n-2}\right) \nabla_{i} u \nabla_{j} u=0, \\
& \Delta u=0,
\end{aligned}
$$

for the Ricci tensor of the base $\left(M^{n}, g_{i j}\right)$. Here $\Delta u:=g^{i j} \nabla_{i} \nabla_{j} u$ is the Laplacian constructed from the connection $\nabla_{i}$ compatible with $g_{i j}$ (note our signature choice for the Laplacian). We note that Equation (1.3) is redundant in that it can be derived from (1.2) using the contracted second Bianchi identity. It is therefore merely an integrability condition for (1.2). 
Definition 1.1. Equations (1.2), (1.3) (or (1.2) alone) are known as the static vacuum Einstein equations. Solutions $g_{i j}$ are called static vacuum metrics.

B List [24, 25], in his Ph.D. thesis under the direction of G. Huisken, presented a system of flow equations whose fixed points solve the static vacuum Einstein equations.

Definition 1.2. List's system of equations is the system

$$
\begin{aligned}
\frac{\partial g_{i j}}{\partial t} & =-2\left(R_{i j}-k_{n}^{2} \nabla_{i} u \nabla_{j} u\right), \\
\frac{\partial u}{\partial t} & =\Delta u .
\end{aligned}
$$

Note that $t$ is the flow parameter, not the time coordinate in the spacetime metric (which we denote by $x^{0}$ ). The metrics $g_{i j}(t ; x)$ are a family of Riemannian metrics on an $n$-manifold, $u(t ; x)$ are a family of functions and $k_{n}$ is an arbitrary constant. When $k_{n}=\sqrt{\frac{n-1}{n-2}}$, the fixed points of List's system are exactly the static vacuum metrics, together with a harmonic function $u$. However, we will keep $k_{n}$ as an arbitrary constant (which obviously can be absorbed in $u$ ) so that we can consider all $n \geq 2$.

A particularly useful equation easily derived from (1.4), (1.5) is

$$
\frac{\partial}{\partial t}|\nabla u|^{2}=\Delta|\nabla u|^{2}-2|\nabla \nabla u|^{2}-2 k_{n}^{2}\left(|\nabla u|^{2}\right)^{2} .
$$

It is now realized that List's system of flow equations is in fact a certain Hamilton-DeTurck flow in one higher dimension; i.e., List's system is really a Ricci flow, modified by pulling back along an evolving diffeomorphism (e.g., [22]). This does not make List's flow any less interesting however. Recall that Hamilton-DeTurck flow is given by

$$
\frac{\partial g_{\mu \nu}}{\partial \lambda}=-2 R_{\mu \nu}+£_{X} g_{\mu \nu}
$$

where $X$ is a vector field. To obtain List's system, choose

$$
\begin{aligned}
g_{\mu \nu} d x^{\mu} d x^{\nu} & =e^{2 k_{n} u} d \tau^{2}+g_{i j} d x^{i} d x^{j}, \\
X & =-\left(g^{i j} \nabla_{i} u\right) \frac{\partial}{\partial x^{j}} .
\end{aligned}
$$

Note that the $g_{\mu \nu}$ in (1.8) differs from that in (1.1). 
It is the purpose of this paper to study the long-time existence properties of solutions of List's system of equations that evolve from a complete, asymptotically flat and rotationally symmetric initial pair $(g(0), u(0))$, subject to the restriction that $g(0)$ does not admit a minimal hypersphere.

It will be convenient to choose a certain coordinate system throughout the flow which will enable us to exploit the initial absence of minimal hyperspheres. This will require that we work with a DeTurck version of List's equations; i.e., that we pull back by a further diffeomorphism on the base manifold. The DeTurck version of equations (1.4) to (1.6) is

$$
\begin{aligned}
\frac{\partial g_{i j}}{\partial t} & =-2\left(R_{i j}-k_{n}^{2} \nabla_{i} u \nabla_{j} u\right)+£_{X} g_{i j} \\
\frac{\partial u}{\partial t} & =\Delta u+£_{X} u \\
\frac{\partial}{\partial t}\left(|\nabla u|^{2}\right) & =\Delta|\nabla u|^{2}-2|\nabla \nabla u|^{2}-2 k_{n}^{2}\left(|\nabla u|^{2}\right)^{2}+£_{X}\left(|\nabla u|^{2}\right)
\end{aligned}
$$

where the vector field $X$ generates the aforementioned diffeomorphism, and it is this system that we will work with directly.

\subsection{Motivations}

List's flow appears as a relatively simple case of the Ricci-harmonic map flow, which has been studied in [27]. The general form of this flow is

$$
\begin{aligned}
& \frac{\partial g_{i j}}{\partial t}=-2 R_{i j}+G_{a b} \nabla_{i} u^{a} \nabla_{j} u^{b}, \\
& \frac{\partial u^{a}}{\partial t}=\Delta u^{a}+g_{i j} \Gamma_{b c}^{a} \nabla^{i} u^{b} \nabla^{j} u^{c},
\end{aligned}
$$

where the $u^{a}$ are embedding functions $u^{a}:\left(M^{n}, g_{i j}\right) \hookrightarrow\left(\mathcal{M}^{m}, G_{a b}\right)$ mapping one Riemannian manifold to another and the $\Gamma_{b c}^{a}$ are the coefficients of the Levi-Cività connection of $G_{a b}$. In the case that $\mathcal{M}^{m}=\mathbb{R}, u^{a}=u$ and $G_{a b}=$ $2 k_{n}^{2}$, these equations reduce to List's flow. Thus, List's flow is the special case of the coupled Ricci-harmonic map flow where the target space $\left(\mathcal{M}^{m}, G_{a b}\right)$ is the real line.

List's flow with rotational symmetry also appears if rotational symmetry is broken in Ricci flow in one more dimension. Rotationally symmetric, asymptotically flat Ricci flow in dimension $n \geq 3$ was studied in connection with a conjecture in string theory regarding the limiting behaviour of Arnowitt-Deser-Misner (ADM) mass as the flow converges [13] (see [21] for an earlier study and see [33] for the $n=2$ case). This is now well-understood, 
at least in the absence of minimal hyperspheres [29] (see also [12]). It is interesting to ask how this understanding is modified if the rotational symmetry is broken down to a subgroup. By the above correspondence between flows, we see that List's flow with rotational symmetry can be thought of as Ricci flow on an $(n+1)$-manifold with $\mathbb{R} \times \mathrm{SO}(n)$ symmetry, which has $\frac{n(n-1)}{2}+1$ generators, or $n-1$ fewer generators than the $\frac{n(n+1)}{2}$ generators of full rotational symmetry in $(n+1)$-dimensions.

However, there is a another reason to study this system, which may prove to be the most compelling. List's equations were conceived as a tool to address conjectures about static metrics in general relativity [20]. We briefly discuss two of these.

We recall Bartnik's quasi-local definition of mass [4]. In an $(n+1)$ dimensional spacetime, consider a moment of time symmetry (i.e., a spacelike hypersurface with zero extrinsic curvature) and in it a bounded $n$-dimensional region $B$. Consider all asymptotically flat Riemannian $n$-manifolds $N$ of non-negative scalar curvature $R \geq 0$ into which $B$ can be isometrically embedded (smoothly in the interior of $B$ ), such that the induced metric and mean curvature must match from both sides of $\partial B$. Further assume that $N$ has no stable minimal sphere lying outside the image of $B$. Then $N$ is called an admissible extension of $B$. By the positive mass theorem $N$ has non-negative ADM mass. Consider all such admissible extensions of $B$ and take the infimum of the ADM masses. This infimum is the Bartnik mass $m_{\mathrm{B}}$ of the region $B$. It is clearly non-negative.

What is not so clear from the definition is whether the mass ever differs from zero. This led Bartnik to make the following conjecture which, if true, would guarantee that the Bartnik mass is nontrivial:

Static minimization conjecture (Bartnik). The infimum is a minimum, and is realized as the ADM mass of a solution of the static vacuum Einstein equations.

Huisken and Ilmanen [19] have since shown by other methods that Bartnik's mass is nonzero except when $B$ is a domain in flat space, thus proving the nontriviality of the Bartnik mass. However the static minimization conjecture has remained open up to now. ${ }^{1}$

\footnotetext{
${ }^{1}$ However, as we were preparing the final draft of this manuscript, a preprint appeared [2] announcing a proof that for any bounded three-dimensional spatial region whose boundary has positive Gauss curvature, there exists an extension satisfying the static Einstein equations with suitable boundary conditions (Bartnik's geometric conditions).
} 
One strategy to address this conjecture may be to choose one admissible, asymptotically flat extension of $B$ and use it as the initial condition for List's flow. Boundary conditions, such as Bartnik's geometric conditions ([5]) that fix the boundary mean curvature and induced metric, must also be imposed at $\partial B$. The idea is then to use the flow to produce a massminimizing sequence which converges to a fixed point, hence to a static metric.

A test case would be to employ this strategy on $\mathbb{R}^{n}$, with no inner boundary. Ideally this would produce sequences of metrics that converge to flat space. ${ }^{2}$ Huisken and Ilmanen [19] have discussed such mass minimizing sequences, and suggest a more complicated view.

Conjecture (Huisken and Ilmanen). Suppose $\left(M, g_{i}\right)$ is a sequence of asymptotically flat, mass-minimizing, non-negative scalar curvature threemetrics tending to zero mass. Then there is a set $Z_{i}$ such that $\left|\partial Z_{i}\right| \rightarrow 0$ and $\left(M \backslash Z_{i}, g_{i}\right)$ has a flat Gromov-Hausdorff limit.

This foresees that an obstruction to convergence may arise in rotationally symmetric, asymptotically flat List flow, in the form of a locally collapsed long, thin tube growing at the origin. ${ }^{3}$

There is some numerical evidence in favour of convergence to flat space. As part of a study motivated by black hole thermodynamics, Headrick and Wiseman [17] examined Ricci flow manifolds-with boundary with $\mathrm{U}(1) \times$ $\mathrm{SO}(3)$ symmetry, including $S^{1} \times \mathbb{B}^{3}$ where $\mathbb{B}^{3}$ denotes a three-ball in $\mathbb{R}^{3}$. They thus had a finitely distant spatial boundary and imposed a Dirichlet condition there. On $S^{1} \times \mathbb{B}^{3}$ they found convergence to flat $S^{1} \times \mathbb{R}^{3}$, known in the physics literature as "hot flat space."

We therefore undertook a study of the long-time existence properties of this flow, with a view to shedding analytical light on these conjectures and numerical results.

\footnotetext{
${ }^{2}$ Note that the manner in which List's flow would produce mass-minimizing sequences will be similar to that of the Ricci flow. There the mass remains constant throughout the flow but will jump to a minimizing value in the limit as $t \rightarrow \infty$, while various measures of the quasi-local mass within bounded regions flow smoothly toward minimizing values $[12,13,29]$.

${ }^{3}$ We expect that if collapse occurred elsewhere, the rotational symmetry would force this to be preceded by formation of a minimal surface. But we will show that the absence of an initial minimal surface implies that none can form later.
} 


\subsection{Overview and main results}

Even with the restriction to rotational symmetry, the above conjectures are not easy to address, and our results are only a starting point. We prove the following:

Theorem 1.3. Let $\left(\tilde{g}_{i j}(r), \tilde{u}(r)\right)$ be asymptotically flat and rotationally symmetric initial data for the system of flow equations (1.10) to (1.12) on $\mathbb{R}^{n}$ such that the metric $\tilde{g}_{i j}(r)$ admits no minimal hypersphere. Then this system of equations has an asymptotically flat, rotationally symmetric solution $\left(g_{i j}(t, r), u(t, r)\right)$ on $\left[0, T_{M}\right) \times[0, \infty)$ for some maximal time of existence $T_{M} \in(0, \infty]$. No minimal hypersphere forms at any $t<\infty$. Furthermore,

(i) If $n=2$, then the flow is immortal $\left(T_{M}=\infty\right)$.

(ii) If $n \geq 3$, and if there is a function $F:[0, \infty) \rightarrow(0, \infty)$ such that $\frac{1}{r}|\nabla u|_{(t, r)} \leq F(t)$, then the flow is immortal.

In the case where the flow fails to exist, we can go some short distance towards analyzing the kind of singularity that develops. List has shown in his thesis [24] that where the flow fails to exist, the norm of the Riemann tensor diverges. We therefore borrow the following definition from the Ricci flow.

Definition 1.4. For $T_{\mathrm{M}}$ the maximal time of existence of the Ricci flow, an essential blow-up sequence $\left(t_{k}, x_{k}\right)$ is a sequence of spacetime points such that $t_{k} \nearrow T_{\mathrm{M}}$ and $\sup _{\left[0, t_{k}\right] \times[0, \infty)}|\operatorname{Riem}|(t, r) \leq C\left|\operatorname{Riem}\left(t_{k}, r_{k}\right)\right|=: B_{k}$ for some constant $C \geq 1$.

Theorem 1.5. Let $(g(t), u(t))$ be a rotationally symmetric solution of (1.10) to (1.12) developing from initial data as in Theorem 1.3, with maximal time of existence $T_{\mathrm{M}}<\infty$, and let $(\bar{g}(t), \bar{u}(t))=\varphi_{t}^{*}(g, u)$ be the corresponding solution of $(1.4,1.5)$. Let $\left(t_{k}, r_{k}\right), t_{k} \nearrow T_{M}$, be an essential blow-up sequence for $(\bar{g}(t), \bar{u}(t))$. Set $B_{k}:=\overline{|\operatorname{Riem}|}\left(t_{k}, r_{k}\right)$ and define the rescalings

$$
\begin{aligned}
g_{(k)(s)} & :=B_{k} \cdot \bar{g}\left(t_{k}+s / B_{k}\right), \\
u_{(k)}(s) & :=\bar{u}\left(t_{k}+s / B_{k}\right), \\
s & \in\left[-B_{k}\left(1+t_{k}\right), 0\right] .
\end{aligned}
$$

Then there is a subsequence of the pointed sequence $\left(\mathbb{R}^{n}, g_{(k)}(s), u_{(k)}(s), r_{k}\right)$ which converges smoothly on all compact subsets of $(-\infty, 0] \times \mathbb{R}^{n}$ to $\left(\mathbb{R}^{n}\right.$, 
$g_{(\infty)}(s)$, const, $\left.r_{\infty}\right)$ with $\left(\mathbb{R}^{n}, g_{(\infty)}(s)\right)$ a complete, ancient solution of the Ricci flow. Sectional curvature in planes tangent to the orbits of the rotational symmetry group is non-negative, and so is the scalar curvature. In $n=3$ dimensions, sectional curvature in planes containing the radial vector is also non-negative.

Theorem 1.5 does not confirm the conjecture above of Huisken-Ilmanen, as it leaves open the possibility that the flow is noncollapsed below some finite scale at the singularity time. When that occurs, then in any dimension, after rescaling, the resulting limit would be noncollapsed below any scale and, in the $n=3$ case, would have non-negative sectional curvatures. By rotational symmetry, the limit would then be a Bryant soliton for $n=3$.

In Section 2 we discuss the notion of asymptotic flatness that we use, and survey results of List on local existence and continuation, making minor modifications where necessary. Section 3 discusses rotational symmetry and its implications. It is in this section that we state the evolution equations in the form that we use and define the basic quantities whose flow we analyse in subsequent sections. Section 4 contains estimates that are valid in arbitrary dimension with no further assumptions beyond rotational symmetry and asymptotic flatness. In Section 5, we assume either that $\frac{1}{r}|\nabla u|$ is bounded on any closed time interval or that the dimension is $n=2$ (in which case it is shown in Section 4 that $\frac{1}{r}|\nabla u|$ is bounded on closed time intervals). Under either of these assumptions, we are then able to obtain all further estimates required to show boundedness of sectional curvatures on finite time intervals. The proofs of Theorems 1.3 and 1.5 then follow easily form these results. These proofs are given in Section 6.

Our sign and index conventions are as follows. As previously stated, we take the (rough or scalar) Laplacian to be $\Delta:=g^{a b} \nabla_{a} \nabla_{b}$. We define the curvature $R_{b c d}^{a} x^{b} y^{c} z^{d}:=\nabla_{y} \nabla_{z} x-\nabla_{z} \nabla_{y} x-\nabla_{[y, z]} x$, and we write $R_{a b c d}:=$ $g_{a e} R_{b c d}^{e}$. The Ricci tensor is $R_{b d}:=R_{b a d}^{a}$. We endeavour where possible to denote constants that bound a function $h$ (say) by $C_{h}^{+}$for an upper bound (i.e., to indicate that $h(t, r) \leq C_{h}^{+}$for all $t$ ) and $C_{h}^{-}$for a lower bound, though we sometimes deviate from this practice for reasons of convenience.

\section{Preliminaries: asymptotic flatness}

The definition of asymptotic flatness, more properly called local asymptotic flatness when $n=2$, can be formulated on any Riemannian manifold with dimension $n \geq 2$ which admits the notion of an asymptotic end. However, since we work on $\mathbb{R}^{n}$, complete generality is not necessary here, though it 
can be achieved with minor changes to the formulation below. On the other hand, our results will hold with a much more general notion of asymptotic flatness for $n \geq 3$ than the usual notion.

To begin, we let

$$
e_{i j}= \begin{cases}\delta_{i j} & \text { for } n \geq 3 \\ \delta_{i j}+a \frac{x_{i} x_{j}}{r^{2}} & \text { for } n=2\end{cases}
$$

where $r=\sqrt{\sum_{i=1}^{n}\left(x^{i}\right)^{2}}$ and, in dimension $n=2, x_{i}=\delta_{i j} x^{j}, a>-1$, and the deficit angle of the flat cone metric is $2 \pi\left(1-\frac{1}{\sqrt{1+a}}\right)$.

Following [24, 25], we define

Definition 2.1. For $n \geq 3,(M, g, u)$ is asymptotically flat (of order one) if there is a compact subset $K \subset M$ such that $M_{K}:=M \backslash K$ is diffeomorphic to $\mathbb{R}^{n} \backslash B_{1}(0)$ where $B_{1}(0)$ is the Euclidean unit ball and, on $M_{K},(g, u)$ satisfies

$$
\begin{aligned}
\left|g_{i j}-e_{i j}\right| & \leq C_{0} / r \\
\left|\partial_{k} g\right| & \leq C_{k} / r^{k+1}, k=1,2,3, \\
|u| & \leq D_{0} / r \\
\left|\partial_{k} u\right| & \leq D_{k} / r^{k+1}, k=1,2,3,
\end{aligned}
$$

where $C_{k}, D_{k}$ are constants $(k \in\{0,1,2,3\}), r^{2}=x_{1}^{2}+\cdots+x_{n}^{2}$ with the $x_{i}$ being Cartesian coordinates for $e_{i j}$, and $\partial_{k}$ is the Cartesian coordinate derivative.

We choose to work in this class for three reasons. The first is that a localin-time existence theorem within this class is already available [24, 25]. The second is that these fall-off conditions are well-suited to the arguments in subsequent sections. The third is that our results are, in fact, not very sensitive to the precise choice of definition of asymptotic flatness. We therefore settle on a convenient choice rather than the most general one, for which the preliminaries would be a greater distraction. ${ }^{4}$

Having said that, we note that when $n>3$ this definition is in fact much weaker than most, which tend to require the difference between the metric

\footnotetext{
${ }^{4}$ For example, a definition based on weighted Sobolev spaces was used for a similar problem in [29]. One can augment that definition by including a condition that $u$ lie in a weighted Sobolev space $H_{\delta}^{k}$ with $k$, the number of derivatives in the Sobolev norm, chosen such that $k>3+n / 2$ and $\delta$, the exponent in the weight factor $r^{\delta}$, any negative number. Then local-in-time existence can be obtained in this class, by modifying the argument in [29] and papers cited therein.
} 
and $e_{i j}$ to decay at $\mathcal{O}\left(1 / r^{n-2}\right)$. However, for $n=3$, the present definition is stronger than necessary.

Proposition 2.2 (List). Let $(\hat{g}, \hat{u})$ be asymptotically flat. Then there exists $a T>0$ such that $(g(t), u(t))$ solves $(1.4,1.5)$ for all $0 \leq t<T$ and $g(0)=\hat{g}$, $u(0)=\hat{u}$. Furthermore, $(g(t), u(t))$ is asymptotically flat for all $0 \leq t<T$.

Proof. See [24], Theorems 3.12 and 9.7, or [25], Theorems 4.1 and 8.6.

Remark 2.3. In fact, List gives a detailed proof of Theorem 2.1 assuming stronger asymptotic flatness conditions and then notes that the proof obviously goes through as well for asymptotic flatness conditions which agree with those above when $n \geq 3$. This is clearly the case, and furthermore it is also the case for $n=2$, with $e_{i j}$ used in place of $\delta_{i j}$ at one step in the proof (Equation (9.7) of [24] or Equation (8.5) of [25]).

It is now possible to state a criterion for the flow to exist for all future time, in the form of a continuation principle which states that, as with Ricci flow, the flow can be continued beyond $t=T$ unless the norm of the Riemann curvature diverges there.

Proposition 2.4. Let $(\hat{g}, \hat{u})$ be a asymptotically flat initial data. Then the system $(1.4,1.5)$, with the initial conditions $g(0)=\hat{g}, u(0)=\hat{u}$ has a unique solution on a maximal time interval $0 \leq t<T_{M} \leq \infty$. If $T_{M}<\infty$ then

$$
\limsup _{t \nearrow T_{M}} \sup _{x \in \mathbb{R}^{n}}|\operatorname{Rm}(t, x)|_{g(t, x)}=\infty
$$

Moreover, for any $T \in\left[0, T_{M}\right)$, if $K=\sup _{0 \leq t \leq T} \sup _{x \in \mathbb{R}^{n}}|\operatorname{Rm}(t, x)|_{g(t, x)}$, and $C=\sup _{x \in \mathbb{R}^{n}}|\nabla \hat{u}(x)|_{g(x)}^{2}$, then

$$
e^{-(2 n K+4 C) T} \hat{g} \leq g(t) \leq e^{(2 n K+4 C) T} \hat{g} \quad \text { for all } t \in[0, T]
$$

Proof. List gives a partial proof for complete manifolds (Theorem 3.22 of [24]) and a full proof for closed manifolds (Theorems 3.11 and 6.22 of [24]). The full proof uses the closedness of the manifold only to invoke the maximum principle for non-negative scalar functions; in particular, norms of $\nabla u$, Riem and derivatives thereof. By Proposition 2.2 and equations (2.2) to (2.5), each such quantity tends to zero as $r \rightarrow \infty, 0 \leq t \leq T$, and thus the maximum principle applies to these functions on complete manifolds as well, provided the initial data obey asymptotic flatness. 


\section{Rotational symmetry}

\subsection{The coordinate system}

Now assume the flow that solves (1.4), (1.5) evolves from rotationally symmetric $C^{2}$ initial data $(\hat{g}, \hat{u})$ with

$$
d \hat{s}^{2}=\hat{g}_{i j} d x^{i} d x^{j}=a^{2}(\rho) d \rho^{2}+\rho^{2} d \Omega^{2},
$$

where $d \Omega^{2}$ is the constant curvature sec $=1$ metric on the $(n-1)$-sphere. We take $a(0)=1, a^{\prime}(0)=0$.

Ricci flow preserves isometries. List's flow, in turn, preserves symmetries of the pair $(g, u)$ (i.e., isometries of $g$ that commute with $u$ ). Combining this fact with Proposition 2.4, then there will be a maximal time of existence $T_{\mathrm{M}} \in(0, \infty]$, a coordinate system in which $(2.1)$ to (2.5) hold, and coordinate transformations taking the metric to a spherical coordinate system $x^{i}=\left(\rho, \theta^{A}\right)$ (with $\theta^{A}$ the coordinates on the $\rho=$ const spheres). In these coordinates, the flow is

$$
\begin{aligned}
t & \mapsto(\bar{g}(t, \rho), \bar{u}(t, \rho)) \\
d \bar{s}^{2} & =\bar{g}_{i j} d x^{i} d x^{j}=q^{2}(t, \rho) d \rho^{2}+h^{2}(t, \rho) d \Omega^{2} .
\end{aligned}
$$

This metric solves (1.4), (1.5). The coordinate functions $q$ and $h$ are $C^{2}$ in $\rho$ and, in the one-sided sense, $C^{1}$ in $t$ at $t=0$ and $\rho>0$, and are smooth in $t$ and $r$ for all $r>0$ and $t \in\left(0, T_{\mathrm{M}}\right)$.

Now introduce a new coordinate system at each time, obtained via acting with the family of diffeomorphisms

$$
\psi_{t}\left(\rho, \theta^{A}\right):=\left(h(t, \rho), \theta^{A}\right)=:\left(r, \theta^{A}\right) .
$$

Note that since $h(0, \rho)=\rho$ then $\psi_{0}=\mathrm{id}$, and also, since $\frac{\partial h}{\partial \rho}(0, \rho)=1$, then for $T$ sufficiently small, there are (possibly $T$ dependent) constants $C_{\partial h}^{ \pm}(T)$ such that

$$
0<C_{\partial h}^{-}(T) \leq \frac{\partial h}{\partial \rho}(t, \rho) \leq C_{\partial h}^{+}(T)
$$

whenever $0 \leq t \leq T$ and $T<\tilde{T}$, where $\tilde{T} \leq T_{\mathrm{M}}$ is defined to be the supremum of $T$-values for which (3.4) is true.

Proposition 3.1. $\tilde{T}=T_{\mathrm{M}}$. 
Proof. Given in Subsection 4.2.2.

We can now write the flow in "area radius gauge" as

$$
\begin{aligned}
t & \mapsto(g(t, r), u(t, r)) \\
g(t, r) & :=\left(\psi_{t}^{-1}\right)^{*} \bar{g}(t, \rho)=f^{2}(t, r) d r^{2}+r^{2} d \Omega^{2}, \\
u(t, r) & :=\left(\psi_{t}^{-1}\right)^{*} \bar{u}(t, \rho)=u(t, h(t, \rho)),
\end{aligned}
$$

where

$$
f(t, r):=\frac{q(t, \rho(t, r))}{\frac{\partial h}{\partial \rho}(t, \rho(t, r))} .
$$

Let the generator of the family $\psi_{t}$ be written as $X^{j}=g^{i j} \nabla_{i} \phi(t, r)$ for some scalar $\phi(t, r)$. Inserting this and (3.5) into (1.10), we obtain the pair of equations

$$
\begin{aligned}
\frac{\partial f}{\partial t} & =-\frac{(n-1)}{r f^{2}} \frac{\partial f}{\partial r}+k_{n}^{2} f|\nabla u|^{2}+\frac{1}{f} \frac{\partial^{2} \phi}{\partial r^{2}}-\frac{1}{f^{2}} \frac{\partial f}{\partial r} \frac{\partial \phi}{\partial r} \\
0 & =\frac{r}{f^{3}} \frac{\partial f}{\partial r}+(n-2)\left(1-\frac{1}{f^{2}}\right)-\frac{r}{f^{2}} \frac{\partial \phi}{\partial r}
\end{aligned}
$$

The latter yields

$$
\frac{\partial \phi}{\partial r}=\frac{1}{f} \frac{\partial f}{\partial r}+\frac{(n-2)}{r}\left(f^{2}-1\right),
$$

We substitute this into (3.7) and (1.11) and define

$$
z:=\frac{1}{f} \frac{\partial u}{\partial r}
$$

so that $z^{2}=|\nabla u|^{2}$ in rotational symmetry. Then Equations (1.10), (1.12) reduce to the system of equations which we study herein, namely:

Definition 3.2. The rotationally symmetric flow equations are the system

$$
\begin{aligned}
\frac{\partial f}{\partial t}= & \frac{1}{f^{2}} \frac{\partial^{2} f}{\partial r^{2}}-\frac{2}{f^{3}}\left(\frac{\partial f}{\partial r}\right)^{2}+\left(\frac{n-2}{r}-\frac{1}{r f^{2}}\right) \frac{\partial f}{\partial r} \\
& -\frac{(n-2)}{r^{2} f}\left(f^{2}-1\right)+k_{n}^{2} f z^{2} \\
\frac{\partial z}{\partial t}= & \frac{1}{f^{2}} \frac{\partial^{2} z}{\partial r^{2}}+\left[\frac{1}{r f^{2}}+\frac{n-2}{r}\right] \frac{\partial z}{\partial r}-\left[\frac{n-1}{r^{2} f^{2}}+k_{n}^{2} z^{2}\right] z .
\end{aligned}
$$


Next, define

$$
\begin{aligned}
\lambda_{1}(t, r) & :=\frac{1}{r f^{3}} \frac{\partial f}{\partial r} \\
\lambda_{2}(t, r) & :=\frac{1}{r^{2}}\left(1-\frac{1}{f^{2}}\right) .
\end{aligned}
$$

Lemma 3.3. When $n=2, \lambda_{1}$ is the Gauss curvature. When $n \geq 3, \lambda_{1}$ is the sectional curvature in planes containing $\frac{\partial}{\partial r}$ and $\lambda_{2}$ is the sectional curvature in planes tangent to the $r=$ const spheres. As well, we have

$$
\begin{aligned}
\mid \text { Riem }\left.\right|^{2} & =R_{i j k l} R^{i j k l}=2(n-1) \lambda_{1}^{2}+(n-1)(n-2) \lambda_{2}^{2}, \\
\frac{\partial \lambda_{2}}{\partial r} & =\frac{2}{r}\left(\lambda_{1}-\lambda_{2}\right) .
\end{aligned}
$$

Proof. The curvature interpretations of $\lambda_{1}$ and $\lambda_{2}$ follow from trivial computations, and then (3.15) follows immediately from rotational symmetry. Equation (3.16) is obvious (expand both sides) and is, in fact, the second Bianchi identity.

Note that (3.16) shows that $\lambda_{1}=\lambda_{2}$ at the origin and, more generally, at any spatial or spacetime extremum of $\lambda_{2}$. Also note that, using Proposition 3.1 and Lemma 3.3, we can adapt the continuation principle (Proposition 2.4) to the area-radius gauge:

Proposition 3.4. If there exists a constant $C_{\lambda}>0$ independent of $T_{M}$ such that

$$
\left|\lambda_{1}(t, r)\right| \leq C_{\lambda} \quad \text { if } n=2,
$$

or

$$
\left|\lambda_{1}(t, r)\right|+\left|\lambda_{2}(t, r)\right| \leq C_{\lambda} \quad \text { if } n \geq 3 \text {, }
$$

for all $(t, r) \in\left[0, T_{\mathrm{M}}\right) \times[0, \infty)$ then $T_{\mathrm{M}}=\infty$.

We shall eventually see that it suffices to bound $\lambda_{1}$ or, equivalently, $R$ from above.

\section{A priori bounds}

In this section and the next, we always assume that $(g(t), u(t)), 0 \leq t \leq T$, is a solution of $(1.10)$ to $(1.12)$ and that $(M, g(t), u(t))$ is asymptotically flat 
(of order one; as in Definition 2.1) for all $t \in[0, T]$. For now $T<\tilde{T} \leq T_{\mathrm{M}}$, but after we prove Proposition 3.1 we will be able to set $\tilde{T}=T_{\mathrm{M}}$.

\subsection{Elementary bounds on scalar quantities}

In his thesis [24, 25], List shows that a modified form of the usual Ricci flow lower bound on scalar curvature of compact manifolds holds for List's flow. He also shows that $|\nabla u|^{2}$ is bounded above by const/(1+t) on a compact manifold. These results are simple applications of the maximum principle. In this section, we adapt the maximum principle to the complete, asymptotically flat setting and obtain bounds on $R$ and $|\nabla u|^{2}$ for asymptotically flat manifolds as corollaries.

Lemma 4.1. Let $\Psi$ be a solution of

$$
\frac{\partial \Psi}{\partial t} \leq \Delta \Psi+\nabla_{Y} \Psi-k^{2} \Psi^{2}
$$

for some vector field $Y$ and constant $k$ on the domain $D(T):=[0, T] \times \mathbb{R}^{n} \ni$ $(t, x)$, such that $\Psi \rightarrow 0$ as $x \rightarrow \infty$. (i) If $\Psi(0, x) \leq 0$ for all $x \in \mathbb{R}^{n}$ then $\Psi(t, x) \leq 0$ for all $(t, x)$ in $D(T)$, and otherwise (ii) if $k \neq 0$ we have $\Psi \leq$ $C_{\Psi}^{+} /(1+t)$ for $C_{\Psi}^{+}:=\max \left\{\frac{1}{k^{2}}, \sup _{x \in \mathbb{R}^{n}} \Psi(0, x)\right\}$.

Proof. Consider the domain $D_{\epsilon}(T):=[0, T] \times B^{n}(1 / \epsilon)$ for $B^{n}(1 / \epsilon)$ the $n$-ball of radius $1 / \epsilon>0$ with respect to the Euclidean metric centred at the origin in $\mathbb{R}^{n}$. To prove (i), we note that (4.1) implies that $\frac{\partial \Psi}{\partial t} \leq \Delta \Psi+\nabla_{Y} \Psi$, and so standard maximum principle arguments show that $\Psi$ must take its maximum on the parabolic boundary of $D_{\epsilon}$ (i.e., points where either $t=0$ or $r=1 / \epsilon)$. Taking $\epsilon \rightarrow 0$, we see by the asymptotic condition on $\Psi$ that either the supremum is 0 or the supremum occurs at $t=0$, in which case it is again zero by assumption.

To prove (ii), we define the function $G:=(1+t) \Psi$ and see that from (4.1) it obeys

$$
\frac{\partial G}{\partial t} \leq \Delta G+\nabla_{Y} G+\frac{G}{1+t}\left[1-k^{2} G\right] .
$$

If the maximum of $G$ on $D_{\epsilon}$ occurs at some $(t, x)$ in the parabolic interior of $D_{\epsilon}$ (i.e., the complement of the parabolic boundary), we see immediately from (4.2) that $G \leq 1 / k^{2}$, and so $\Psi \leq \frac{1}{k^{2}(1+t)}$. If instead the maximum of $G$ occurs on the boundary of $B^{n}(1 / \epsilon)$ at some $0<t<T$, then by taking $\epsilon$ 
small enough we see that the maximum approaches zero. Alternatively, the maximum can occur at $t=0$, and since $G(0, x)=\Psi(0, x)$ then in this case $\Psi(t, x) \leq \sup _{x} \frac{G(0, x)}{1+t}=\sup _{x} \frac{\Psi(0, x)}{1+t}$.

This immediately yields Propositions 4.2 and 4.4 .

Proposition 4.2. Let $(g, u)$ be an asymptotically flat solution of the flow on the domain $[0, T] \times \mathbb{R}^{n} .0<T<\tilde{T}$ Then for any $n \geq 2$

$$
|\nabla u(t, r)| \leq \frac{1}{\sqrt{1+t}} C_{|\nabla u|}^{+}
$$

where $C_{|\nabla u|}^{+}$is a constant depending only on the initial data and $k_{n}$.

Proof. Equation (1.12) yields

$$
\frac{\partial}{\partial t}\left(|\nabla u|^{2}\right) \leq \Delta\left(|\nabla u|^{2}\right)+\nabla_{X}\left(|\nabla u|^{2}\right)-2 k_{n}^{2}\left(|\nabla u|^{2}\right)^{2} .
$$

Apply Lemma 4.1 with $\Psi=|\nabla u|^{2}$ and $k^{2}=2 k_{n}^{2}$. This proves the proposition and shows that $C_{|\nabla u|}^{+}=\max \left\{\frac{1}{\sqrt{2 k_{n}^{2}}}, \sup _{x}|\nabla u|_{(0, x)}\right\}$.

Remark 4.3. In rotational symmetry, we have $|z|=\left|\frac{1}{f} \frac{\partial u}{\partial r}\right|=|\nabla u|$ and we write this bound as

$$
|z| \leq \frac{C_{z}^{+}}{\sqrt{1+t}}
$$

where $C_{z}^{+}:=C_{|\nabla u|}^{+}$.

Next, from (1.10) it is easy to derive (see [24, Ch. 2]) that

$$
\frac{\partial R}{\partial t}=\Delta R+2 R_{i j} R^{i j}+2 k_{n}^{2}(\Delta u)^{2}-2 k_{n}^{2}|\nabla \nabla u|^{2}-4 k_{n}^{2} R_{i j} \nabla^{i} u \nabla^{j} u+\nabla_{X} R .
$$

Unlike in the Ricci flow, this equation does not lead to the preservation of scalar curvature. However, defining

$$
S_{i j}:=R_{i j}-k_{n}^{2} \nabla_{i} u \nabla_{j} u
$$


and using (1.12), then (4.1) leads to

$$
\begin{aligned}
\frac{\partial S}{\partial t} & =\Delta S+2 S_{i j} S^{i j}+2 k_{n}^{2}(\Delta u)^{2}+\nabla_{X} S \\
& =\Delta S+2\left(S_{i j}-\frac{1}{n} g_{i j} S\right)\left(S^{i j}-\frac{1}{n} g^{i j} S\right)+\frac{2}{n} S^{2}+2 k_{2}^{n}(\Delta u)^{2}+\nabla_{X} S \\
(4.8) & \geq \Delta S+\frac{2}{n} S^{2}+\nabla_{X} S,
\end{aligned}
$$

where $S:=g^{i j} S_{i j}$. Then we obtain

Proposition 4.4. Let $(g, u)$ be an asymptotically flat solution of the flow on the domain $[0, T] \times \mathbb{R}^{n}, 0<T<\tilde{T}$. For any $n \geq 2$

$$
S:=R-k_{n}^{2}|\nabla u|^{2} \geq \frac{C_{S}^{-}}{1+t},
$$

where $C_{S}^{-} \leq 0$ is a constant depending only on the initial data and $n$, and if $S(0, x) \geq 0$ for all $x \in \mathbb{R}^{n}$, then $S(t, x) \geq 0$ for all $t \geq 0$ and all $x \in \mathbb{R}^{n}$.

Proof. Use (4.8) to apply Lemma 4.1 to $-S$. The $k^{2}$ of Lemma 4.1 takes the value $-2 / n$. This yields $C_{S}^{-}=\min \left\{-\frac{n}{2}, \inf _{x} S(0, x)\right\}$.

Note that List's flow does not necessarily preserve positive scalar curvature, though it does preserve the positivity of $R-k_{n}^{2}|\nabla u|^{2}$.

\subsection{Bounds that hold in rotational symmetry}

The bounds of the previous subsection are valid with or without rotational symmetry. We now specialize to the rotationally symmetric flow equations (3.11), $(3.12)$ on $[0, T] \times[0, \infty) \ni(t, r), 0<T<\tilde{T}$.

4.2.1. Bounds on $f$. In this subsection, we derive bounds on $f:=\sqrt{g_{r r}}$. These bounds allow us to address two concerns. The first is that our coordinate system may break down during the flow. This will happen if $f$ diverges to $+\infty$ or approaches zero along the flow. Note that $f$ diverges at some $r>0$ iff the mean curvature $H$ of the $r=$ const sphere goes to zero. The mean curvature is given by

$$
H=\frac{n-1}{r f} .
$$

Thus, divergence of $f$ at finite $r$ implies the presence or formation of a minimal hypersphere. We will show that this cannot happen. 
The second concern arises because positivity of the scalar curvature is not strictly preserved along the flow, even though the results of the previous subsection show that $R$ is bounded below and the bound tends to zero in time. One may wonder whether this is enough for purposes of the static minimization conjecture, where one seems to want the static metric to arise as a limit of a sequence of positive scalar curvature metrics.

Here we show that the rotationally symmetric flow $(3.11),(3.12)$ on $\mathbb{R}^{3}$ does preserve the positivity of the Brown-York and Misner-Sharpe quasilocal masses. The Brown-York mass of a closed embedded hypersurface $\Sigma \hookrightarrow \mathbb{R}^{3}$ is defined to be $\mu_{\mathrm{BY}}[\Sigma]:=\int_{\Sigma}\left(H_{0}-H\right) d \Sigma$, where $H$ is the mean curvature of $\Sigma$ and $H_{0}$ is the mean curvature of an isometrically embedded image of $\Sigma$ in flat space. In our case, for a sphere of radius $r$ about the origin, we have

$$
\mu_{\mathrm{BY}}[\Sigma]:=\frac{8 \pi}{r}\left(1-\frac{1}{f(t, r)}\right) .
$$

The Misner-Sharpe mass is defined only for rotationally symmetric metrics and is given for $n=3$ by

$$
\mu_{\mathrm{MS}}:=\frac{8 \pi}{r}\left(1-\frac{1}{f^{2}(t, r)}\right)=\left(1+\frac{1}{f}\right) \mu_{\mathrm{BY}}
$$

so it is positive if and only if the Brown-York mass is.

We now show that for any finite $t$ along the flow, $f(t, r)$ is bounded above and below. As a result, if no minimal sphere is present initially then none will form, and for $n=3$ if $\mu_{\mathrm{BY}}$ is initially positive then it will always be so (likewise for $\mu_{\mathrm{MS}}$ ). In fact, this will hold in any dimension if we take (4.11) and (4.12), without modification, to be the definitions of $\mu_{\mathrm{BY}}$ and $\mu_{\mathrm{MS}}$ in any dimension (this is not what is usually done, however).

\section{Proposition 4.5.}

$$
C_{f}^{-} \leq f(t, r) \leq C_{f}^{+}(1+t)^{p}
$$

where $p=1+\left(k_{n} C_{z}^{+}\right)^{2}$ for all $n \geq 2$. The constants $C_{f}^{ \pm}$depend only on the initial data $\{(f(0, r), z(0, r))\}$ and, for $n=2, f_{\infty}$ (equivalently, the $n=2$ mass). 
Proof. Let $w(t, r)=f^{2}(t, r)-1$. Then (3.11) yields

$\frac{\partial w}{\partial t}=\frac{1}{f^{2}} \frac{\partial^{2} w}{\partial r^{2}}-\frac{3}{2 f^{4}}\left(\frac{\partial w}{\partial r}\right)^{2}+\left(\frac{n-2}{r}-\frac{1}{r f^{2}}\right) \frac{\partial w}{\partial r}-\frac{2(n-2)}{r^{2}} w+2 k_{n}^{2} f^{2} z^{2}$,

subject to the boundary conditions

$$
w(t, r) \rightarrow \begin{cases}0 & \text { for } r \rightarrow 0 \text { and all } n \geq 2, \\ w_{\infty}:=f_{\infty}^{2}-1 & \text { for } r \rightarrow \infty \text { and } n=2, \text { and } \\ 0, & \text { for } r \rightarrow \infty \text { and } n>2\end{cases}
$$

Now consider the closed annular region $A_{\epsilon}(T):=[0, T] \times\left[\epsilon, \frac{1}{\epsilon}\right] \ni(t, r)$.

(i) Case $n>2$ : By (4.15), $\inf \{w(t, r) \mid 0 \leq t \leq T, r \geq 0\} \leq 0$. We observe from (4.14) that if $w(t, r)$ has a negative minimum in $A_{\epsilon}(T)$, such a minimum must lie on the parabolic boundary. ${ }^{5}$ Taking $\epsilon$ sufficiently large, then by the boundary conditions, the negative minimum must lie on the initial boundary, so then $w(t, r) \geq \inf _{r}\{w(0, r)\}$.

(ii) Case $n=2$ : We consider the function $W_{\epsilon}(t, r)=w(t, r)+\epsilon \cdot t$ on $A_{\epsilon}(T)$. From (4.14), $W$ has the following evolution equation:

$$
\frac{\partial W_{\epsilon}}{\partial t}=\frac{1}{f^{2}} \frac{\partial^{2} W_{\epsilon}}{\partial r^{2}}-\frac{3}{2 f^{4}}\left(\frac{\partial W_{\epsilon}}{\partial t}\right)^{2}-\frac{1}{r f^{2}} \frac{\partial W_{\epsilon}}{\partial r}+2 k_{n}^{2} f^{2} z^{2}+\epsilon,
$$

with boundary conditions $W_{\epsilon}(t, r) \rightarrow \epsilon \cdot t$ as $r \rightarrow 0$ and $W_{\epsilon}(t, r) \rightarrow$ $w_{\infty}+\epsilon \cdot t$ as $r \rightarrow \infty$. For $\delta>0$, the minimum of $W_{\epsilon}$ on $A_{\epsilon}(T)$ must lie on the parabolic boundary. Taking $\epsilon \rightarrow 0$, then we obtain $w(t, r) \geq$ $\min \left\{0, w_{\infty}, \inf _{r}\{w(0, r)\}\right\}$.

This proves the left-hand (i.e., inferior) inequality in (4.13) and shows that

$$
C_{f}^{-}= \begin{cases}\inf _{r}\{f(0, r)\} & \text { for } n>2, \\ \min \left\{1, f_{\infty}, \inf _{r}\{f(0, r)\}\right\} & \text { for } n=2 .\end{cases}
$$

To prove the superior inequality, consider the function

$$
Q(t, r):=\frac{w(t, r)}{(1+t)^{2 p}} .
$$

\footnotetext{
${ }^{5}$ e.g., Let $\Psi=-w$ and then observe that the inequality (4.1) applies on $A_{\epsilon}(T)$ (with $k=0$ ), so we can use Lemma 4.1(i).
} 
For $t<\tau, Q$ obeys $Q(0, r)=w(0, r), Q(t, 0)=0$, and either $\lim _{r \rightarrow \infty} Q(t, r)=$ 0 if $n>2$ or $\lim _{r \rightarrow \infty} Q(t, r)=\frac{w_{\infty}}{(1+t)^{2 p}}$ if $n=2$. As well, $Q$ solves the PDE

$$
\begin{aligned}
\frac{\partial Q}{\partial t}= & \frac{1}{f^{2}} \frac{\partial^{2} Q}{\partial r^{2}}-\frac{3(1+t)^{2 p}}{2 f^{4}}\left(\frac{\partial Q}{\partial r}\right)^{2}+\left(\frac{n-2}{r}-\frac{1}{r f^{2}}\right) \frac{\partial Q}{\partial r} \\
& +\left(2 k_{n}^{2} z^{2}-\frac{2(n-2)}{r^{2}}-\frac{2 p}{1+t}\right) Q+\frac{2 k_{n}^{2}}{(1+t)^{2 p}} z^{2} \\
\leq & \frac{1}{f^{2}} \frac{\partial^{2} Q}{\partial r^{2}}-\frac{3(1+t)^{2 p}}{2 f^{4}}\left(\frac{\partial Q}{\partial r}\right)^{2}+\left(\frac{n-2}{r}-\frac{1}{r f^{2}}\right) \frac{\partial Q}{\partial r} \\
& +\left(\frac{2 k_{n}^{2}\left(C_{z}^{+}\right)^{2}}{1+t}-\frac{2(n-2)}{r^{2}}-\frac{2 p}{1+t}\right) Q+\frac{2 k_{n}^{2}\left(C_{z}^{+}\right)^{2}}{(1+t)^{2 p+1}}
\end{aligned}
$$

using (4.5). Choose

$$
p:=1+\left(k_{n} C_{z}^{+}\right)^{2}
$$

Then (4.19) yields

$$
\frac{\partial Q}{\partial t} \leq \frac{1}{f^{2}} \frac{\partial^{2} Q}{\partial r^{2}}+\left(\frac{n-2}{r}-\frac{1}{r f^{2}}\right) \frac{\partial Q}{\partial r}+\frac{2}{1+t}\left(\frac{\left(k_{n} C_{z}^{+}\right)^{2}}{(1+t)^{2 p}}-Q\right)
$$

whenever $Q \geq 0$, where we have discarded some manifestly negative terms. Clearly this equation does not permit $Q$ to have a maximum on the parabolic interior of $[0, T] \times[\epsilon, 1 / \epsilon]$ unless $Q \leq \frac{\left(k_{n} C_{z}^{+}\right)^{2}}{(1+t)^{2 p}}$, whence by (4.18) we get $w(t, r) \leq\left(k_{n} C_{z}^{+}\right)^{2}$ and then

$$
f(t, r) \leq \sqrt{1+\left(k_{n} C_{z}^{+}\right)^{2}} .
$$

Otherwise, the maximum of $Q$ can occur on the parabolic boundary. Then taking $\epsilon$ sufficiently small, if a positive maximum for $Q$ occurs either:

(a) The maximum of $Q$ occurs on the initial boundary $t=0$. This can occur for any $n \geq 2$. Using (4.18), $w=f^{2}-1$, and the fact that $Q(0, r)=$ $w(0, r)=f^{2}(0, r)-1$, then $f^{2}(t, r) \leq 1+(1+t)^{2 p} \sup _{r}\left\{f^{2}(0, r)-1\right\} \leq$ $(1+t)^{2 p} \sup _{r}\left\{f^{2}(0, r)\right\}$. Combining this with (4.22) yields

$$
\begin{aligned}
& f(t, r) \leq \max \left\{\sqrt{1+\left(k_{n} C_{z}^{+}\right)^{2}},(1+t)^{p} \sup _{r}\{f(0, r)\}\right\} \leq C_{f}^{+}(1+t)^{p} \\
& \text { for } C_{f}^{+}=\max \left\{\sqrt{1+\left(k_{n} C_{z}^{+}\right)^{2}}, \sup _{r}\{f(0, r)\}\right\}, \text { or }
\end{aligned}
$$


(b) $n=2$ and the maximum of $Q$ is $\frac{w_{\infty}}{(1+t)^{2 p}}<\max \left\{0, w_{\infty}\right\}$. Combining this with (4.22) and (4.23), we obtain

$$
f(t, r) \leq \max \left\{f_{\infty}, \sqrt{1+\left(k_{n} C_{z}^{+}\right)^{2}},(1+t)^{p} \sup _{r}\{f(0, r)\}\right\} \leq C_{f}^{+}(1+t)^{p},
$$

for $C_{f}^{+}=\max \left\{f_{\infty}, \sqrt{1+\left(k_{n} C_{z}^{+}\right)^{2}}, \sup _{r}\{f(0, r)\}\right\}$.

Corollary 4.6. (i) If no minimal hypersphere is present initially, none forms at any $t<\infty$. (ii) For $n=2$, if the Brown-York mass $\mu_{\mathrm{BY}}(0, r)$ of every $r=$ const hypersphere about the origin is $\geq 0$ at $t=0$, then $\mu_{\mathrm{BY}}(t, r) \geq$ 0 for every $r \in \mathbb{R}$ and every $t>0$; the same holds for the Misner-Sharpe mass.

Proof. The first statement follows immediately from (4.23) and (4.10). The second statement follows from (4.17), (4.13) and (4.11) (or, for the MisnerSharpe mass, (4.12)).

Remark 4.7. If the assumptions of (ii) hold and if the flow converges $\left(\mathbb{R}^{3}, g(t), 0\right)$ in the pointed Cheeger-Gromov sense to $\left(\mathbb{R}^{3}, g_{\infty}, 0\right)$, then $\left(\mathbb{R}^{3}\right.$, $\left.g_{\infty}\right)$ will have non-negative Brown-York mass at each $r$ (by (4.11) and the fact that the sign of $1-\frac{1}{f}$ will be preserved under the diffeomorphisms $\left.\left(\mathbb{R}^{3}, g(t), 0\right) \rightarrow\left(\mathbb{R}^{3}, g_{\infty}, 0\right)\right)$. Since $\lim _{r} \mu_{\mathrm{BY}}=m_{\mathrm{ADM}}:=\frac{1}{16 \pi} \int_{S_{\infty}^{2}} \delta^{i j}\left(g_{k i, j}-\right.$ $\left.g_{i j, k}\right) d S^{i}$ (we take this limit along $r=$ const $\rightarrow \infty$ spheres), the ADM mass of the limit manifold will be non-negative.

\subsubsection{Proof of Proposition 3.1}

Proof. Setting $t=0$ in (4.13), we see that $C_{f}^{-} \leq f(0, r) \equiv a(r) \equiv q(0, \rho(r)) \leq$ $C_{f}^{+}$. Assume, by way of contradiction, that $\tilde{T}<T_{\mathrm{M}}$. Then, by Proposition 2.4, there are constants $K$ and $C$ such that

$$
e^{-(2 n K+4 C) T}\left(C_{f}^{-}\right)^{2} \leq q^{2}(t, \rho) \leq e^{(2 n K+4 C) T}\left(C_{f}^{+}\right)^{2}
$$

for $0 \leq t \leq T$. Furthermore, (4.13) holds for all $t \in[0, T]$ so, dividing (4.25) by (4.13) and using (3.6), we get

$$
e^{-(2 n K+4 C) T}\left(\frac{C_{f}^{-}}{C_{f}^{+}(1+T)^{p}}\right)^{2} \leq \frac{q^{2}(t, \rho(r))}{f^{2}(t, r)}=\left(\frac{\partial h}{\partial \rho}\right)^{2} \leq e^{(2 n K+4 C) T}\left(\frac{C_{f}^{+}}{C_{f}^{-}}\right)^{2}
$$


for all $t \in[0, T]$. We can replace $T$ using that $T \leq \tilde{T}<T_{\mathrm{M}}$, obtaining

$$
e^{-(2 n K+4 C) T_{M}}\left(\frac{C_{f}^{-}}{C_{f}^{+}\left(1+T_{M}\right)^{p}}\right)^{2} \leq\left(\frac{\partial h}{\partial \rho}\right)^{2} \leq e^{(2 n K+4 C) T_{M}}\left(\frac{C_{f}^{+}}{C_{f}^{-}}\right)^{2}
$$

By comparison, we see that the constants in the inequality (3.4) are in fact independent of $T$. Since the inequalities hold for any $T<\tilde{T}$ and are closed relations, they hold for $T=\tilde{T}$ as well and, by adjusting the constants slightly if necessary (keeping the inferior one positive of course), then (3.4) holds for $t$-values beyond $\tilde{T}$, contradicting the assumption.

4.2.3. A bound on tangential sectional curvature. We will now obtain a bound on the behaviour of $f$ at the origin. This is in fact a lower bound on $\lambda_{2}$, which for $n \geq 3$ is the sectional curvature in planes tangent to the $r=$ const spheres.

Proposition 4.8. For all $n \geq 2, \lambda_{2}(t, r)$ is bounded below by a constant $-C_{\lambda_{2}}^{-} \leq 0$ which depends only on the initial data $f(0, r)$ such that $\lambda_{2}(t, r) \geq$ $-C_{\lambda_{2}}^{-} /(1+t)$.

Proof. In close (but not exact) analogy to [29], we will approximate $\lambda_{2}$ by a sequence of functions $u_{m}(t, r), 0<m<2$, defined by

$$
\begin{aligned}
& u_{m}(t, r):=\left(\frac{2}{r^{m}+r^{2}}\right)\left(1-\frac{1}{f^{2}}\right) \text { for } r>0, \\
& u_{m}(t, 0):=\lim _{r \rightarrow 0} u_{m}(t, r) .
\end{aligned}
$$

The $u_{m}(t, r)$ functions have the following useful properties:

(i) $u_{m}(t, 0)=0$ for all $0<m<2$ and $\lim _{r \rightarrow \infty} u_{m}(t, r)=0$ for all $0<m \leq 2$.

(ii) For fixed $t$ and $r \neq 0$, the map $m \mapsto u_{m}(t, r)$ is continuous at $m=2$, and in fact

$$
\lambda_{2}=u_{2}
$$


Now define new functions

$$
U_{m}(t, r)=(1+t) u_{m}(t, r)
$$

and note that $U_{m}(0, r)=u_{m}(0, r)$. From (3.11) we obtain an evolution equation for $U_{m}(t, r)$ given by

$$
\begin{aligned}
\frac{\partial U_{m}}{\partial t}= & \frac{1}{f^{2}} \frac{\partial^{2} U_{m}}{\partial r^{2}}+\frac{\left(r^{m}+r^{2}\right)}{4(1+t)}\left(\frac{\partial U_{m}}{\partial r}\right)^{2}+\frac{\left(2 r+m r^{m-1}\right)}{2(1+t)} U_{m} \frac{\partial U_{m}}{\partial r} \\
& +\left[\frac{2\left(2 r+m r^{m-1}\right)}{f^{2}\left(r^{m}+r^{2}\right)}-\frac{1}{r f^{2}}+\frac{(n-2)}{r}\right] \frac{\partial U_{m}}{\partial r} \\
& +\frac{1}{2\left(1+r^{2-m}\right)(1+t)}\left[(4-m)(m+n-2)+m(n-2)+2(n-1) r^{2-m}\right. \\
& \left.+r^{m-2}(2-m)(m+n-2)+r^{m-2} m\left(\frac{m}{2}+n-2\right)\right] U_{m}^{2} \\
& -\frac{(2-m)(m+n-2)}{r^{2}\left(1+r^{2-m}\right)} U_{m}+\left(\frac{4(1+t)}{r^{m}+r^{2}}\right) k_{n}^{2} z^{2}
\end{aligned}
$$

$$
\begin{aligned}
-2 k_{n}^{2} z^{2} U_{m} & +\frac{1}{(1+t)} U_{m} \\
\geq \frac{1}{f^{2}} \frac{\partial^{2} U_{m}}{\partial r^{2}}+ & {\left[\frac{\left(2 r+m r^{m-1}\right)}{2(1+t)} U_{m}+\frac{2\left(2 r+m r^{m-1}\right)}{f^{2}\left(r^{m}+r^{2}\right)}\right.} \\
& \left.-\frac{1}{r f^{2}}+\frac{(n-2)}{r}\right] \frac{\partial U_{m}}{\partial r}
\end{aligned}
$$

$$
+\frac{1}{1+t}\left[(n-1) U_{m}^{2}+U_{m}\right]-\frac{(2-m)(m+n-2)}{r^{2}\left(1+r^{2-m}\right)} U_{m}-2 k_{n}^{2} z^{2} U_{m},
$$

where the inequality holds at least for $1 \leq m<2$ and $n \geq 2$. Furthermore, if

$$
U_{m}<-\frac{1}{n-1}
$$

we then obtain

$$
\begin{aligned}
\frac{\partial U_{m}}{\partial t}> & \frac{1}{f^{2}} \frac{\partial^{2} U_{m}}{\partial r^{2}}+\left[\frac{\left(2 r+m r^{m-1}\right)}{2(1+t)} U_{m}+\frac{2\left(2 r+m r^{m-1}\right)}{f^{2}\left(r^{m}+r^{2}\right)}\right. \\
& \left.-\frac{1}{r f^{2}}+\frac{(n-2)}{r}\right] \frac{\partial U_{m}}{\partial r}
\end{aligned}
$$


As with Proposition 4.5, we work first on the annulus $A_{\epsilon}(T)$. From (4.34) and (4.35), we see that $U_{m}$ cannot have a minimum $<-\frac{1}{n-1}$ at some $\left(t_{0}, r_{0}\right)$ in the parabolic interior of the annulus. The minimum, if $<-\frac{1}{n-1}$, must lie on the parabolic boundary of $A_{\epsilon}(T)$. Taking $\epsilon \rightarrow 0$ and recalling that $u_{m}(t, \epsilon) \rightarrow 0$ and $u_{m}(t, 1 / \epsilon) \rightarrow 0$, whence $U_{m}(t, \epsilon) \rightarrow 0$ and $U_{m}(t, 1 / \epsilon) \rightarrow 0$ as well, then the minimum, if $<-\frac{1}{n-1}$, must lie at $t=0$; that is,

$$
\begin{aligned}
U_{m}(t, r) & \geq \min \left\{-\frac{1}{n-1}, \inf _{r}\left\{U_{m}(0, r)\right\}\right\}=\min \left\{-\frac{1}{n-1}, \inf _{r}\left\{u_{m}(0, r)\right\}\right\} \\
& =\min \left\{-\frac{1}{n-1}, \inf _{r}\left\{\frac{2}{r^{m}+r^{2}}\left(1-\frac{1}{f^{2}(0, t)}\right)\right\}\right\} \\
& \geq \min \left\{-\frac{1}{n-1}, \inf _{r}\left\{\frac{2}{r^{2}}\left(1-\frac{1}{f^{2}(0, t)}\right)\right\}\right\} \\
(4.36) \quad & =\min \left\{-\frac{1}{n-1}, 2 \inf _{r}\left\{\lambda_{2}(0, r)\right\}\right\}=:-C_{\lambda_{2}}^{-},
\end{aligned}
$$

where $C_{\lambda_{2}}^{-} \geq 0$. We now take $m \nearrow 2$ in (4.36), so that $U_{m} \rightarrow(1+t) \lambda_{2}$ by (4.30) and (4.31). Using (4.34) and (4.35) as well, (4.36) yields

$$
\lambda_{2}(t, r) \geq-\frac{C_{\lambda_{2}}^{-}}{(1+t)},
$$

\subsubsection{Smoothness of $|\nabla u|$ for $n=2$}

Proposition 4.9. Assume $n=2$. Then

$$
\frac{1}{r}|\nabla u(t, r)| \leq C_{\zeta}^{+}
$$

where the constant $C_{\zeta}^{+}$depends only on the (smooth) initial data for $\nabla u$.

Proof. Let $\zeta_{m}(t, r)=2 \frac{z(t, r)}{r+r^{m}}$ for $0<m<1$. Computing from (3.12) we then obtain that $\zeta_{m}$ obeys

$$
\begin{aligned}
\frac{\partial \zeta_{m}}{\partial t}= & \frac{1}{f^{2}} \frac{\partial^{2} \zeta_{m}}{\partial r^{2}}+\left\{\frac{3+(2 m+1) r^{m-1}}{r f^{2}\left(1+r^{m-1}\right)}+\frac{(n-2)}{r}\right\} \frac{\partial \zeta_{m}}{\partial r} \\
& +\frac{(m-1) r^{m-1}}{r^{2}\left(1+r^{m-1}\right)}\left\{\frac{m+1}{f^{2}}+(n-2)\right\} \zeta_{m} \\
& +(n-2) \lambda_{2} \zeta_{m}-k_{n}^{2} z^{2} \zeta_{m} .
\end{aligned}
$$


For $\zeta_{m}>0, m<1$, and $n=2,(4.39)$ reduces to

$$
\frac{\partial \zeta_{m}}{\partial t} \leq \frac{1}{f^{2}} \frac{\partial^{2} \zeta_{m}}{\partial r^{2}}+\left[\frac{3+(2 m+1) r^{m-1}}{r f^{2}\left(1+r^{m-1}\right)}\right] \frac{\partial \zeta_{m}}{\partial r} .
$$

As usual, restrict attention to the annulus $A_{\epsilon}(T):=[0, T] \times[\epsilon, 1 / \epsilon]$, for some chosen $\epsilon>0$ and $T<\tau, \tau$ as above. By the maximum principle, $\zeta_{m}$ must have a maximum in $A_{\epsilon}(T)$, but by (4.40) this cannot occur in the parabolic interior of $A_{\epsilon}(T)$. If the maximum occurs at $r=\epsilon$, then take $\epsilon \rightarrow 0$. By regularity, $\frac{\partial u}{\partial r} \in \mathcal{O}(r)$ as $r \rightarrow 0$, so $\zeta_{m}(t, \epsilon) \in \mathcal{O}\left(\epsilon^{1-m}\right) \rightarrow 0, \forall t \in[0, T]$. Similarly, at large $r, \frac{\partial u}{\partial r} \in \mathcal{O}(1 / r)$ and so $\zeta_{m} \rightarrow 0$ as $r=1 / \epsilon \rightarrow \infty$. Thus, for $\epsilon$ small enough, $\zeta_{m}$ cannot have a positive maximum at any $t>0$, and since the supremum of $\zeta_{m}$ is non-negative on the $t=0$ boundary then

$$
\left|\zeta_{m}(t, r)\right| \leq \sup _{r}\left\{\left|\zeta_{m}(0, r)\right|\right\} \leq \sup _{r}\left\{\frac{2}{r}|z(0, r)|\right\}=: C_{\zeta}^{+}
$$

where the smoothness of the initial data is used to infer the boundedness of $|z(0, r)| / r$. Finally, since $C_{\zeta}^{+}$is independent of $m$, we can take $m \nearrow 1$ to complete the argument.

\subsection{Summary of a priori bounds}

In summary, we have the following bounds for all $t \in[0, T]$, for all $x \in \mathbb{R}^{n}$, and, assuming rotational symmetry, for all $r \in[0, \infty)$.

1. const $\leq f^{2} \leq$ const $\cdot(1+t)^{p}$.

2. $R(t, x) \geq-\frac{\text { const }}{1+t}$.

3. $R(t, x) \geq k_{n}^{2}|\nabla u|^{2}$ for all $(t, x)$ if it holds at $t=0$.

4. $\lambda_{2}(t, r) \geq-\frac{\text { const }}{(1+t)}$ and $\lambda_{2}(t, r) \geq 0$ if $f(0, r) \geq 1$ for all $r$.

5. $|\nabla u|^{2} \leq \frac{\text { const }}{1+t}$.

6. $\frac{1}{r}|\nabla u| \leq$ const when $n=2$.

The constants denoted const here are positive and distinct. These constants, and $p$, depend only on the initial data, $n, k_{n}$ and (for $n=2$ ) $f_{\infty}$, and do not depend on $T$, as can be seen by the explicit expressions for the constants given in the preceding section.

For $n \geq 3$, we can summarize the picture that these bounds present as follows. 
Proposition 4.10. Assume $n \geq 3$. One of the following possibilities holds:

1. The flow (3.12), (3.13) exists for all $(t, r) \in[0, \infty) \times[0, \infty)$.

2. There is a sequence of points $\left(t_{k}, r_{k}\right)$ with $r_{k} \rightarrow 0$ such that $\lambda_{1}\left(t_{k}, r_{k}\right)=$ $\lambda_{2}\left(t_{k}, r_{k}\right) \rightarrow+\infty$ as $t_{k} \nearrow T_{\mathrm{M}}$.

3. There is a sequence of points $\left(t_{k}, r_{k}\right)$ along which $\lambda_{1}\left(t_{k}, r_{k}\right) \rightarrow+\infty$ as $t_{k} \nearrow T_{\mathrm{M}}$ but $\lambda_{2}\left(t_{k}, r_{k}\right)$ remains bounded along every such sequence.

Remark 4.11. The considerations of the next section will eliminate the third possibility from this list.

Corollary 4.12. Either the flow exists for all $t>0$ or $\lim \sup _{t} T_{\mathrm{M}} \sup _{r}$ $\lambda_{1}=\infty$ and $\lim \sup _{t} T_{\mathrm{M}} \sup _{r} R=\infty$.

Remark 4.13. The Corollary is also true for $n=2$, since then $2 \lambda_{1}=$ $R \geq-\frac{\text { const }}{1+t}$.

Proof of 4.10. By the continuation principle, either the flow exists for all $t>$ 0 or at least one sectional curvature diverges as $t \rightarrow T_{\mathrm{M}}$. We first consider the case of $\lambda_{2} \rightarrow \infty$. Then there is a sequence of points $\left(t_{k}, r_{k}\right), t_{k}<t_{k+1}<T_{\mathrm{M}}$, along which $\lambda_{2}$ assumes successive maximum values; $\lambda_{2}\left(t_{k}, r_{k}\right) \geq \lambda_{2}(t, r)$ for all $(t, r) \in\left[0, t_{k}\right] \times[0, \infty)$. From the definition of $\lambda_{2}$, we see that $r_{k} \rightarrow 0$ along any such sequence, and at these points, the Bianchi identity (3.16) shows that $\lambda_{1}\left(t_{k}, r_{k}\right)=\lambda_{2}\left(t_{k}, r_{k}\right)$.

Since $\lambda_{2} \geq-\frac{\text { const }}{1+t}$, the only remaining cases are those for which $\lambda_{2}$ remains bounded. But then $\lambda_{1}$ cannot diverge to $-\infty$ because $(n-1)\left(2 \lambda_{1}+\right.$ $\left.(n-2) \lambda_{2}\right) \equiv R \geq-\frac{\text { const }}{1+t}$. Thus we have eliminated all possibilities that are not enumerated in the proposition.

\section{Smoothness of $|\nabla u|$ and long-time existence}

\subsection{Smoothness of $|\nabla u|$ and an upper bound on $\lambda_{2}$}

In this section, we will show that whenever $\frac{1}{r}|\nabla u|$ remains finite, the flow exists for all $t>0$. When $n=2$, we have already shown in Proposition 4.9 that $\frac{1}{r}|\nabla u|$ remains bounded. In this subsection, the first proposition we present shows that this will also be the case for $n>3$, provided that $\lambda_{2}$ remains finite. In consequence, the $n \geq 3$ flow will fail to exist only if $\lambda_{2}$ diverges at finite $T$. We then show that, conversely, when $\frac{1}{r}|\nabla u|$ remains finite, so does $\lambda_{2}$. This follows for all dimensions, including $n=2$, and is 
useful when $n=2$ even though the combination $\lambda_{2}=\frac{1}{r^{2}}\left(1-\frac{1}{f^{2}}\right)$ is of course not a sectional curvature in that case.

Proposition 5.1. Assume that there is a function $F_{\lambda_{2}}^{+}:[0, \infty) \rightarrow[0, \infty)$ such that $\lambda_{2}(t, r) \leq F_{\lambda_{2}}^{+}(T)$ for all $0 \leq t \leq T$. Then there is a function $F_{\zeta}^{+}$: $[0, \infty) \rightarrow[0, \infty)$ such that

$$
\frac{z}{r} \equiv \frac{1}{r}|\nabla u(t, r)| \leq F_{\zeta}^{+}(T) \text { whenever } 0 \leq t \leq T .
$$

Proof. By Proposition 4.9, this is true for $n=2$ (without the assumption on $\lambda_{2}$ and with $F_{\zeta}^{+}=C_{\zeta}^{+}=$const). Thus, assume $n \geq 3$. Choose some $T>0$ and define $\xi_{m}:=\zeta_{m} /(1+t)^{(n-2) F_{\lambda_{2}}^{+}(T)}$ for $0 \leq t \leq T$. Then from (4.39) with $0<m<1$ and $\xi_{m}>0$, we obtain

$$
\frac{\partial \xi_{m}}{\partial t} \leq \frac{1}{f^{2}} \frac{\partial^{2} \xi_{m}}{\partial r^{2}}+\left[\frac{3+(2 m+1) r^{m-1}}{r f^{2}\left(1+r^{m-1}\right)}\right] \frac{\partial \xi_{m}}{\partial r},
$$

and the proof proceeds precisely as in Proposition 4.9. This implies that $\xi_{m}$ is bounded above by a constant depending only on initial data, and thus $\zeta_{m} \leq F_{\zeta}^{+}(T):=$ const $\cdot(1+t)^{(n-2) F_{\lambda_{2}}^{+}(T)}$. Since $F_{\lambda_{2}}^{+}$is defined for all $T>0$, so is $F_{\zeta}^{+}$, and since the bound is $m$-independent, we extend to $m=1$.

Proposition 5.2. Conversely, assume that Equation (5.1) holds for all $T>0$. Then there is a function $F_{\lambda_{2}}^{+}:[0, \infty) \times[0, \infty)$ such that

$$
\lambda_{2}(t, r) \leq F_{\lambda_{2}}^{+}(T) \text { whenever } 0 \leq t \leq T \text {. }
$$

Proof. We work, as always, on a compact annular domain $A_{\epsilon}(T):=[0, T] \times$ $[\epsilon, 1 / \epsilon] \ni(t, r)$. Choose a positive function $F:[0, \infty) \rightarrow(0, \infty)$ such that $F(0)=1$ and define functions

$$
\begin{aligned}
V_{m}(t, r) & :=\left(\frac{F(t)}{r^{m}+r^{2}}\right)\left(f^{2}(t, r)-1\right) \quad \text { for } r>0, \\
V_{m}(t, 0) & :=\lim _{r \rightarrow 0} V_{m}(t, r) .
\end{aligned}
$$

Note that

$$
\lambda_{2}=\frac{2}{F(t) f^{2}(t, r)} V_{2}
$$

We will show by the maximum principle that the $V_{m}(t, r)$ functions have a uniform bound in $m$. From (3.11), we obtain an evolution equation for 
$V_{m}(t, r)$ given by

$$
\begin{aligned}
\frac{\partial V_{m}}{\partial t}= & \frac{1}{f^{2}} \frac{\partial^{2} V_{m}}{\partial r^{2}}+\left[\frac{2\left(m r^{m-1}+2 r\right)}{f^{2}\left(r^{m}+r^{2}\right)}-\frac{3\left(r^{m}+r^{2}\right)}{2 f^{4} F} \frac{\partial V_{m}}{\partial r}\right. \\
& \left.-\frac{3\left(m r^{m-1}+2 r\right)}{f^{4} F} V_{m}+\frac{n-2}{r}-\frac{1}{r f^{2}}\right] \frac{\partial V_{m}}{\partial r} \\
& +\frac{r^{m-2}}{r^{m}+r^{2}}\left[\frac{m(m-2)}{f^{2}} V_{m}+(n-2)(m-2) V_{m}+2 k_{n}^{2}|\nabla u|^{2} r^{2-m} F\right] \\
& +\left[2 k_{n}^{2}|\nabla u|^{2}+\frac{F^{\prime}}{F}\right] V_{m}-\frac{3\left(m r^{m-1}+2 r\right)^{2}}{2 f^{4} F\left(r^{m}+r^{2}\right)} V_{m}^{2} .
\end{aligned}
$$

If $V_{m}(t, r)$ attains a positive maximum $V_{m}\left(t_{0}, r_{0}\right)<1$ for all $m<2$, we are done, so assume to the contrary that the maximum is $>1$. As well, for the moment assume that the maximum occurs at a point $\left(t_{0}, r_{0}\right)$ in the parabolic interior of $A_{\epsilon}(T)$. All the terms in (5.7) that do not contain a derivative will be negative provided that

$$
\frac{2 k_{n}^{2}|\nabla u|_{0}^{2}}{r_{0}^{m}+r_{0}^{2}} F\left(t_{0}\right)+2 k_{n}^{2}|\nabla u|_{0}^{2} V_{m}\left(t_{0}, r_{0}\right)+\frac{F^{\prime}\left(t_{0}\right)}{F\left(t_{0}\right)} V_{m}\left(t_{0}, r_{0}\right) \leq 0 .
$$

Observe that this implies that $F^{\prime}\left(t_{0}\right)<0$, so take it to be decreasing for all $t \geq 0$. Then $0<F(t) \leq 1$ and so (5.8) will hold if it holds with $F\left(t_{0}\right)$ replaced by 1 in the first term on the left. Then, in the limit as $m \nearrow 2$, this first term becomes $k_{n}^{2}\left(\frac{1}{r}|\nabla u|_{0}\right)^{2}$. Since we assume that (5.1) holds, we can control this term. Then we obtain the sufficient condition

$$
2 k_{n}^{2}\left(F_{\zeta}^{+}(T)\right)^{2}+\left[2 k_{n}^{2}\left(C_{z}^{+}\right)^{2}+\frac{F^{\prime}\left(t_{0}\right)}{F\left(t_{0}\right)}\right] V_{m}\left(t_{0}, r_{0}\right) \leq 0 .
$$

Since $V\left(t_{0}, r_{0}\right) \geq 1$ by assumption, a choice of $F$ that satisfies this condition is

$$
F(t)=e^{-P t}, P:=2 k_{n}^{2}\left[\left(F_{\zeta}^{+}(T)\right)^{2}+\left(C_{z}^{+}\right)^{2}\right] .
$$

That is, choosing $F$ in (5.4) to be given by (5.10), then either (i) $V_{m}$ is bounded above on $A_{\epsilon}(T)$ by 1 or the maximum of $V_{m}$ on $A_{\epsilon}(T)$ resides on the parabolic boundary of $A_{\epsilon}(T)$. On the spatial part of this boundary at $r_{0}=1 / \epsilon$, as $\epsilon \rightarrow 0$ we see from (5.4), (5.6), and asymptotic flatness that $V_{m} \rightarrow 0$, so for any fixed $\epsilon$ small enough, if the maximum were to occur on this part of the boundary it would be less than 1. Likewise, if it occurs at $r_{0}=\epsilon$, then from (5.4) we would have $V_{m}\left(t_{0}, \epsilon\right) \sim F\left(t_{0}\right)\left(f^{2}-1\right) / \epsilon^{m}, m<2$, 
and then local existence implies $V_{m}\left(t_{0}, \epsilon\right) \rightarrow 0$ as $\epsilon \rightarrow 0$, so again for any fixed but sufficiently small $\epsilon$ we would have $V_{m}<1$ at its maximum. Thus, the maximum, if $>1$, occurs on the initial boundary, and so

$$
\begin{aligned}
V_{m}(t, r) & \leq \max \left\{1, \sup _{r}\left\{V_{m}(0, r)\right\}\right\} \leq \max \left\{1, \sup _{r}\left\{2 V_{2}(0, r)\right\}\right\} \\
& =\max \left\{1, \sup _{r}\left\{2 \lambda_{2}(0, r)\right\}\right\},
\end{aligned}
$$

for any $m<2$. Since the right-hand side is independent of $m<2$, the proposition now follows by taking $m \nearrow 2$ and using (5.10), (5.6) and the inferior part of (4.13). We note that we obtain $F_{\lambda_{2}}^{+}(T) \leq$ const $\cdot e^{P T}$ with $P$ as in (5.10).

It immediately follows that the other sectional curvature, $\lambda_{1}$, is bounded below:

Corollary 5.3. Assume that (5.1) holds for all $T>0$. Then there is a function $F_{\lambda_{1}}^{-}:[0, \infty) \times[0, \infty)$ such that

$$
\lambda_{1}(t, r) \geq-F_{\lambda_{1}}^{-}(T) \text { whenever } 0 \leq t \leq T \text {. }
$$

Proof. In rotational symmetry, we have

$$
R=2(n-1) \lambda_{1}+(n-1)(n-2) \lambda_{2} .
$$

The result then follows from (4.9) and Proposition 5.2, and indeed $F_{\lambda_{1}}^{-}(T) \leq$ const $\cdot e^{P T}$ with $P$ as in (5.10).

\subsection{Bounding the Hessian of $u$}

Finally we seek an upper bound for $\lambda_{1}$. To find it, we must first bound the second $r$-derivative of $u$.

Proposition 5.4. Assume that (5.1) holds for all $T>0$. Then there is a function $F_{\left|z^{\prime}\right|}^{+}:[0, \infty) \times[0, \infty)$ such that

$$
\left|z^{\prime}(t, r)\right| \leq F_{\left|z^{\prime}\right|}^{+}(T) \text { whenever } 0 \leq t \leq T \text {. }
$$

Proof. Recall that in rotational symmetry we have $|z|=\left|\frac{1}{f} \frac{\partial u}{\partial r}\right|=|\nabla u|$, with $\left|z^{\prime}\right|=\left|\frac{\partial}{\partial r}\left(\frac{1}{f} \frac{\partial u}{\partial r}\right)\right|=\frac{\partial}{\partial r}|\nabla u|$. The evolution equation for $\left(z^{\prime}\right)^{2}$ can be derived 
from (3.11), (3.12) and is given by

$$
\begin{aligned}
\frac{\partial}{\partial t}\left[\left(z^{\prime}\right)^{2}\right]= & \frac{1}{f^{2}} \frac{\partial^{2}}{\partial r^{2}}\left[\left(z^{\prime}\right)^{2}\right]+\left(\frac{1}{r f^{2}}+\frac{n-2}{r}-\frac{2}{f^{3}} \frac{\partial f}{\partial r}\right) \frac{\partial}{\partial r}\left[\left(z^{\prime}\right)^{2}\right] \\
& -\frac{2}{f^{2}}\left(\frac{\partial z^{\prime}}{\partial r}\right)^{2}-2\left(R+\frac{n(n-1)}{r^{2} f^{2}}\right)\left(\frac{\left(z^{\prime}\right)^{2}}{n-1}+\frac{z}{r} z^{\prime}\right) \\
& -6 k_{n}^{2} z^{2}\left(z^{\prime}\right)^{2}-\frac{2}{r^{2}}(n-1)(n-2)\left(\frac{\left(z^{\prime}\right)^{2}}{(n-1) f^{2}}-\frac{z}{r} z^{\prime}\right)
\end{aligned}
$$

Set $0 \leq t \leq T$ and define

$$
\mathcal{Z}:=e^{-2 \kappa t}\left(z^{\prime}\right)^{2}
$$

where the constant $\kappa>0$ will be chosen below. Then from (5.15) we compute that

$$
\begin{aligned}
\frac{\partial \mathcal{Z}}{\partial t}= & \frac{1}{f^{2}} \frac{\partial^{2} \mathcal{Z}}{\partial r^{2}}+\left(\frac{1}{r f^{2}}+\frac{n-2}{r}-\frac{2}{f^{3}} \frac{\partial f}{\partial r}\right) \frac{\partial \mathcal{Z}}{\partial r}-\frac{1}{2 f^{2} \mathcal{Z}}\left(\frac{\partial \mathcal{Z}}{\partial r}\right)^{2} \\
& -2\left(R+\frac{n(n-1)}{r^{2} f^{2}}\right)\left[\frac{\mathcal{Z}}{n-1}+\frac{z}{r} e^{-\kappa t} \sqrt{\mathcal{Z}}\right] \\
& -\frac{2}{r^{2}}(n-1)(n-2)\left[\frac{\mathcal{Z}}{(n-1) f^{2}}-\frac{z}{r} e^{-\kappa t} \sqrt{\mathcal{Z}}\right]-2\left[3 k_{n}^{2} z^{2}+\kappa\right] \mathcal{Z}
\end{aligned}
$$

By asymptotic flatness, $\mathcal{Z} \rightarrow 0$ as $r \rightarrow \infty$ for an $t<T$. Since $\mathcal{Z} \geq 0$ by definition, either $\mathcal{Z}=z^{\prime}=0$ everywhere or $\mathcal{Z}$ has a positive maximum. Say the maximum of $\mathcal{Z}$ occurs at a spacetime point $q=(t, r)$.

(i) Case $R=: R_{q} \geq 0$ at $q$ and $r(q) \neq 0$ : From (5.17) it follows that at $q$ we must have

$$
\begin{aligned}
0 \leq \frac{\partial \mathcal{Z}}{\partial t} \leq & -2\left[R_{q}+\frac{n(n-1)}{r^{2} f^{2}}\right]\left[\frac{\mathcal{Z}}{n-1}+\frac{z}{r} e^{-\kappa t} \sqrt{\mathcal{Z}}\right] \\
& -\frac{2}{r^{2}}(n-1)(n-2)\left[\frac{\mathcal{Z}}{(n-1) f^{2}}-\frac{z}{r} e^{-\kappa t} \sqrt{\mathcal{Z}}\right]
\end{aligned}
$$

and so we must have that

$$
\sqrt{\mathcal{Z}} \leq-(n-1) \frac{z}{r} e^{-\kappa t} \leq(n-1) F_{\zeta}^{+}(T) e^{-\kappa t}
$$


if $n=2$, and if $n>2$ then either (5.19) must hold or

$$
\sqrt{\mathcal{Z}} \leq(n-1) f^{2} \frac{z}{r} e^{-\kappa t} \leq F_{\zeta}^{+}(T)\left(C_{f}^{+}\right)^{2}(1+t)^{2 p} e^{-\kappa t}
$$

must hold instead, using (5.1) and (4.13) of Proposition 4.5 (where $p$ is defined) and (4.5). In either case, $\mathcal{Z}$ and, thus, $z^{\prime}$ are bounded above at any $t \geq 0$.

(ii) Case $R<0$ at $q$ and $r(q) \neq 0$ : We further assume that neither (5.19) nor (5.20) holds at the maximum, since otherwise we would have an upper bound on $\mathcal{Z}$. Then the terms in square brackets in (5.17) are non-negative so where they are multiplied by negative coefficients in (5.17) we can drop them and obtain that

$$
0 \leq \frac{\partial \mathcal{Z}}{\partial t} \leq-2 R_{q}\left(\frac{\mathcal{Z}}{n-1}+\frac{z}{r} e^{-\kappa t} \sqrt{\mathcal{Z}}\right)-2 \kappa \mathcal{Z}
$$

at the point $q$ where $\mathcal{Z}$ takes its maximum. Choose $\kappa$ such that $\kappa+$ $\frac{R_{q}}{n-1}>0$. For example, choose

$$
\kappa=1+\frac{\left|C_{S}^{-}\right|}{n-1}
$$

where $C_{S}^{-}$is a lower bound for $R(\operatorname{cf}(4.9))$. Then (5.21) yields

$$
\sqrt{\mathcal{Z}} \leq-R_{q} \frac{z}{r} e^{-\kappa t} \leq\left|C_{s}^{-}\right| F_{\zeta}^{+}(T) e^{-\kappa t}
$$

(iii) Maximum occurs at $r=r(q)=0$ : By local existence, $f$ and $z$ and their spatial derivatives are bounded for $0 \leq t \leq T$, for any $T<\tau=$ maximal time of existence. Thus the same is true for $\mathcal{Z}$ and for its first time derivative. Examining the behaviour of coefficients in (5.17) as $r \rightarrow 0$, keeping in mind that $z / r$ is bounded, we see that this implies that

$$
\frac{n}{f^{2}}\left[\frac{\mathcal{Z}}{n-1}+\frac{z}{r} e^{-\kappa t} \sqrt{\mathcal{Z}}\right]+(n-2)\left[\frac{\mathcal{Z}}{(n-1) f^{2}}-\frac{z}{r} e^{-\kappa t} \sqrt{\mathcal{Z}}\right] \in \mathcal{O}\left(r^{2}\right)
$$

as $r \rightarrow 0$ for all $0 \leq t \leq T$. Taking the limit as $r \rightarrow 0$ of (5.24), we obtain either

$$
\sqrt{\mathcal{Z}(t, 0)}=0
$$


or

$$
\sqrt{\mathcal{Z}(t, 0)}=\frac{1}{2} f^{2}\left(n-2-\frac{n}{f^{2}}\right) \frac{z}{r} e^{-\kappa t} .
$$

(iv) Maximum occurs at $t=0$ : Then from (5.16) we get

$$
\sqrt{\mathcal{Z}(0, r)} \equiv\left|z^{\prime}(0, t)\right| \leq \text { const }
$$

since the initial data for $z^{\prime}$ is bounded.

At least one of the bounds given by (5.19), (5.20), (5.23), (5.25) to (5.27) must hold and so, using the definition (5.16) with $\kappa$ given by (5.22), we obtain (5.14).

\subsection{An upper bound on transverse sectional curvature}

With the Hessian bound in hand, the following estimate then gives the desired upper bound for $\lambda_{1}$.

Proposition 5.5. Assume that (5.1) holds for all $T>0$. Then there is a function $F_{\lambda_{1}}^{+}:[0, \infty) \times[0, \infty)$ such that

$$
\lambda_{1}(t, r) \leq F_{\lambda_{1}}^{+}(T) \text { whenever } 0 \leq t \leq T
$$

Proof. We first define

$$
y(t, r):= \begin{cases}\frac{1}{2} r \frac{\partial}{\partial r}\left[\frac{1}{r^{2}}\left(\frac{1}{f}-1\right)\right] & \text { for } r>0 \\ 0 & \text { for } r=0\end{cases}
$$

and we note that

$$
y=\frac{f}{(1+f)} \lambda_{2}-\frac{1}{2} f \lambda_{1} \text { for } r>0
$$

so we seek a lower bound for $y$. To see that $y$ is continuous at $r=0$, use the Bianchi identity (3.16) to write (5.30) as

$$
y=-\frac{r f}{2(1+f)} \frac{\partial \lambda_{2}}{\partial r}+\frac{f(1-f)}{2(1+f)} \lambda_{1} .
$$

Since $1-f \in \mathcal{O}\left(r^{2}\right)$ and $\frac{\partial \lambda_{2}}{\partial r} \in \mathcal{O}(r)$ as $r \rightarrow 0$, we see that $y \rightarrow 0$ as $r \rightarrow 0$. 
Computing from (3.11) and (5.29), we see that for $r>0 y$ obeys

$$
\begin{aligned}
\frac{\partial y}{\partial t}= & \frac{1}{f^{2}} \frac{\partial^{2} y}{\partial r^{2}}+\left(\frac{\alpha}{r}\right) \frac{\partial y}{\partial r}+\frac{8}{f} y^{2}+\frac{1}{r^{2}}[\beta y+\gamma] \\
& -k_{n}^{2} z^{2} y+k_{n}^{2} \frac{z^{2}}{r^{2}}-\frac{k_{n}^{2}}{f} \frac{z}{r} z^{\prime} \\
\alpha:= & \frac{4 r^{2}}{f} y+\frac{5}{f^{2}}-\frac{4}{f}+n-2 \\
\beta:= & \frac{4}{f^{2}}-\frac{8}{f}+(n-2)\left(1-\frac{3}{f^{2}}\right), \\
\gamma:= & \frac{(n-2)}{r^{2}}\left(1-\frac{1}{f}\right)^{3} .
\end{aligned}
$$

Using the definition of $\lambda_{2}$, we simplify $\beta$ and $\gamma$ as follows:

$$
\begin{aligned}
\frac{\beta}{r^{2}} & =\left(n-2-\frac{4}{(1+f)}\right) \lambda_{2}-\frac{4}{r^{2} f}-\frac{2(n-2)}{r^{2} f^{2}} \\
& \leq\left(n-2-\frac{4}{(1+f)}\right) \lambda_{2}, \\
\frac{\gamma}{r^{2}} & =(n-2) \frac{f(f-1)}{(f+1)^{2}} \lambda_{2}^{2} \geq-\frac{(n-2) f}{(f+1)^{2}} \lambda_{2}^{2} .
\end{aligned}
$$

Then whenever $y<0$ and $r>0$ we have that (5.32) yields

$$
\begin{aligned}
\frac{\partial y}{\partial t} \geq & \frac{1}{f^{2}} \frac{\partial^{2} y}{\partial r^{2}}+\left(\frac{\alpha}{r}\right) \frac{\partial y}{\partial r}+\frac{8}{f} y^{2}+\left(n-2-\frac{4}{(1+f)}\right) \lambda_{2} y \\
& -\frac{(n-2) f}{(1+f)^{2}} \lambda_{2}^{2}-\frac{k_{n}^{2}}{f} \frac{z}{r} z^{\prime}
\end{aligned}
$$

In particular, we work as usual on the domain $A_{\epsilon}(T):=[0, T] \times\left[\epsilon, \frac{1}{\epsilon}\right]$ and then observe immediately from (5.38) that, if $y$ takes its minimum in the parabolic interior, then $y(t, r)$ is bounded below by

$$
\begin{aligned}
y(t, r) \geq- & \frac{1}{2}\left(n-2-\frac{4}{(1+f)}\right) \frac{f \lambda_{2}}{8} \\
\text { 39) }- & {\left[\left(n-2-\frac{4}{(1+f)}\right)^{2} \frac{f^{2} \lambda_{2}^{2}}{64}+\frac{(n-2) f^{2}}{8(1+f)^{2}} \lambda_{2}^{2}+\frac{k_{n}^{2}}{8} \frac{z}{r} z^{\prime}\right]^{1 / 2}, }
\end{aligned}
$$

and then the bounds (4.13), (4.37), (5.1), (5.3) and (5.14) on the quantities appearing on the right-hand side prove the proposition. 
On the other hand, $y$ could have its minimum on the parabolic boundary of $A_{\epsilon}(T)$. If the minimum occurs at $t=0$, then $y$ is bounded below by $\min \left\{0, \inf _{r}\{y(0, r)\}\right\}$, again proving the proposition. If, however, the minimum occurs at $r=\epsilon$ or $r=1 / \epsilon$, then if we choose $\epsilon$ small enough this minimum would approach zero (since $y(t, \epsilon) \rightarrow 0$ as $r \rightarrow 0$ by the argument at the start of the proof, and $y(t, 1 / \epsilon) \rightarrow 0$ by asymptotic flatness, as seen from, say, (5.30)).

\section{Proofs of Theorems 1.3 and $\mathbf{1 . 5}$}

Proof of 1.3. By assumption, there is no minimal hypersphere at $t=0$. By (4.10) and (4.13), then no minimal hypersphere can form at any $t \in\left[0, T_{\mathrm{M}}\right)$.

Furthermore, $\lambda_{2}$ is bounded below for all $t>0$ (Proposition 4.8). Now assume that Equation (5.1) holds. Then for all $t>0, \lambda_{2}$ is bounded above (Proposition 5.1) and $\lambda_{1}$ is bounded below (Corollary 5.3) and above (Proposition 5.5). Thus the maximal time of existence is $T_{\mathrm{M}}=\infty$ (Proposition 3.4).

In particular, if $n=2$, then (5.1) holds (Proposition 4.9).

Proof of 1.5. There are no closed geodesics of $g(t)$, for if there were then by rotational symmetry such a geodesic would necessarily lie on a minimal hypersphere, and we have shown that there are none of those. Then by standard results ([32], paragraph 6.6.1), the injectivity radius of the manifold at time $t$ will be equal to the conjugate radius and thus bounded below by $\pi / \sqrt{\sup _{r}|\operatorname{Riem}(t, r)|}$. These facts are diffeomorphism invariant and so apply equally to $\bar{g}(t)$ (see $(3.2,3.5)$ ). Choosing an essential blow-up sequence for $\bar{g}, \bar{u})$ and rescaling as in $(1.15)$, then along each rescaled flow $\left(g_{(k)}, u_{(k)}\right)$ the injectivity radius of $g_{(k)}(s)$ is uniformly (in $s$ and in $k$ ) bounded below by $\pi / \sqrt{C}$.

In view of Equation (1.9), define $X_{(k)}^{i}:=-g_{(k)}^{i j} \nabla^{(k)} u_{(k)}$. Note that

$$
\begin{aligned}
\left|X_{(k)}\right|^{2} & :=\left[g_{(k)}^{i j} \nabla_{i}^{(k)} u_{(k)} \nabla_{j}^{(k)} u_{(k)}\right]_{s}=\left[\frac{1}{B_{k}} \bar{g}^{i j} \bar{\nabla}_{i} \bar{u} \bar{\nabla}_{j} \bar{u}\right]_{\left(t_{k}+s / B_{k}\right)} \\
& \leq \frac{\mathrm{const}}{B_{k}\left(1+t_{k}+s / B_{k}\right)} \\
& \rightarrow 0
\end{aligned}
$$

In particular, for each $k$ the diffeomorphisms generated by $X_{(k)}$ are defined (and, indeed, getting smaller). Thus, we can use the correspondence between List's flow and Ricci flow (1.7) to (1.9) to express the sequence $\left(g_{(k)}(s)\right.$, 
$\left.u_{(k)}(s)\right)$ as a sequence of Ricci flows $G_{(k)}(s)$ in $(n+1)$-dimensions. Because $\frac{\partial}{\partial \tau}(\mathrm{cf}(1.8))$ is a Killing vector field, the injectivity radius remains bounded below by $\pi / \sqrt{C}$. Let $x_{k}:=\left(\tau_{k}, r_{k}\right)$ and choose $\tau_{k}=0$ (since $\frac{\partial}{\partial \tau}$ is a Killing vector field, the choice is irrelevant). By a theorem of Hamilton [14], the pointed sequence $\left(M, G_{(k)}(s), x_{k}\right)$ converges to a complete pointed Ricci flow $(M, G(s), x)$. The domain of $s$ is the limit of the intervals $\left[-B_{k}\left(1+t_{k}\right), 0\right]$ and is thus $(-\infty, 0]$, so $G$ is an ancient solution of Ricci flow. The injectivity radius of $(M, G(s))$ is bounded below (uniformly in $s)$ at $x$.

We see from (6.1) that $u_{(\infty)}$ is constant in $r$. It is constant in $\tau=x^{0}$ by assumption and then is constant in the flow time $s$ (equivalently, in $t)$ as well by the asymptotic condition $u(t, r) \rightarrow$ const as $r \rightarrow \infty$. It follows that the Ricci flow for the limit metric $G(s)$ in $(n+1)$-dimensions is trivial in the $\tau$ direction, and splits as an ancient Ricci flow for $g$ (the induced metric for $\tau=0$ ) in $n$-dimensions, together with the equation $u=$ const.

Since $\lambda_{2}(t) \geq-$ const for the flow (Prop 4.8) of $(g, u)$ (and thus for the unrescaled flow of $(\bar{g}, \bar{u})$ since the condition is natural with respect to diffeomorphisms) and since rescaling divides $\lambda_{2}$ by the maximum of the norm of the curvature, the limit of rescaled flows is a flow with $\lambda_{2}(s) \geq 0$. That is, the limit flow has non-negative sectional curvature in planes tangent to the orbits of symmetry. By Theorem 2.4 of [8], any ancient, complete, threedimensional solution of Ricci flow has non-negative sectional curvatures in all planes, thus including radial planes as well as tangential planes when $n=3$. Chen also observed that any ancient, complete flow has $R \geq 0$, but here we can see this directly by the same argument as with $\lambda_{2}$, since $R \geq-$ const along the original flow (Proposition 4.4).

\section{Acknowledgments}

This work was carried out during visits of TAO to the University of Alberta, EW to Monash University, and all three authors to the Banff International Research Station. TAO and EW also thank the Arnold Sommerfeld Centre of the LMU Munich and the organizers of the Workshop on Field Theory and Geometric Flows. EW thanks the Ennio di Giorgi Centre, Scuola Normale Superiore di Pisa, and the organizers of the Pisa workshop on Geometric Flows in Mathematics and Theoretical Physics. We thank Maria Athanassenas and Reto Müller for discussions. The work was partially supported by a Discovery Grant to EW from the Natural Sciences and Engineering Research Council of Canada. 


\section{References}

[1] M.T. Anderson, On the structure of solutions to the static vacuum Einstein equations, Ann. Henri Poincaré 1 (2000), 995-1042.

[2] M.T. Anderson and M. Khuri, The static extension problem in general relativity, Preprint, 2009.

[3] R. Bartnik, The mass of an asymptotically flat manifold, Comm. Pure Appl. Math. 39 (1986), 661-693.

[4] R. Bartnik, New definition of quasilocal mass, Phys. Rev. Lett. 62 (1989), 2346-2348; Mass and 3-metrics of non-negative scalar curvature, Proc. Int. Congress Math., Beijing, 2002, 2, 231-240 [arXiv:math/0304259]; Energy in general relativity, in 'Tsing Hua lectures on geometry and analysis', ed. S.-T. Yau, International Press, Cambridge MA, USA, 1995, 5-27.

[5] R. Bartnik, seminar given at BIRS Workshop on Geometric Flows in Mathematics and Physics (Banff, 13-18 April 2008), unpublished.

[6] S. Brendle and R.M. Schoen, Classification of manifolds with $\frac{1}{4}$-pinched curvature, Acta Math. 200 (2008), 1-13; Manifolds with $\frac{1}{4}$-pinched curvature are space forms, J. Amer. Math. Soc. 22 (2009), 287-307.

[7] J.D. Brown and J.W. York, Quasilocal energy and conserved charges derived from the gravitational action, Phys. Rev. D (3) 47 (1993), 1407-1419.

[8] B.-L. Chen, Strong uniqueness of the Ricci flow, J. Differential Geom. 82 (2009), 363-382 [arxiv:0706.3081].

[9] B. Chow, S.-C. Chu, D. Glickenstein, C. Guenther, J. Isenberg, T. Ivey, D. Knopf, P. Lu, F. Luo and L. Ni, The Ricci flow: techniques and applications, Part I: Geometric Applications, Mathematical Surveys and Monographs, 135, American Mathematical Society, Providence, RI, 2007.

[10] B. Chow, P. Lu and L. Ni, Hamilton's Ricci Flow, Graduate Studies in Mathematics, 77, American Mathematical Society, Providence, RI, 2006 .

[11] H.-D. Cao and X.-P. Zhu, Hamilton-Perelman's proof of the Poincaré conjecture and the geometrization conjecture, Asian J. Math. 10 (2006), 169-492. 
[12] X. Dai and L. Ma, Mass under the Ricci flow, Comm. Math. Phys. 274 (2007), 65-80.

[13] M. Gutperle, M. Headrick, S. Minwalla and V. Schomerus, Space-time energy decreases under world sheet RG flow, JHEP 0301 (2003), 073 [arxiv:hep-th/0211063].

[14] R.S. Hamilton, A compactness property for solutions of the Ricci flow, Amer. J. Math. 117 (1995), 545-572.

[15] S. Harris, Conformally stationary spacetimes, Classical Quantum Gravity 9 (1992), 1823-1827.

[16] S.W. Hawking and G.F.R. Ellis, The large scale structure of space-time, Cambridge Univeristy Press, Cambridge, 1973.

[17] M. Headrick and T. Wiseman, Ricci flow and black holes, Classical Quantum Gravity 23 (2006), 6683-6708.

[18] G. Huisken and T. Ilmanen, The Riemannian Penrose inequality, Int. Math. Res. Not. 20 (1997), 1045-1058.

[19] G. Huisken and T. Ilmanen, The inverse mean curvature flow and the Riemannian Penrose inequality, J. Differential Geom. 59 (2001), 353-437.

[20] G Huisken, private communication.

[21] T.A. Ivey, The Ricci flow on radially symmetric $\mathbb{R}^{3}$, Comm. Partial Differential Equations 19 (1994), 1481-1500.

[22] J.-F. Li, unpublished notes; for details, see M.M. Akbar and E. Woolgar, Ricci solitons and Einstein-Scalar field theory, Classical Quantum Gravity 26 (2009), 055015 [arxiv:0808.3126].

[23] A. Lichnerowicz, Théories rélativistes de la gravitation et de l'électromagnétisme, Masson, Paris, 1955.

[24] B. List, Evolution of an extended Ricci flow system, Ph.D. thesis 2005, Freie Universität Berlin, unpublished.

[25] B. List, Evolution of an extended Ricci flow system, Comm. Anal. Geom. 16 (2008), 1007-1048.

[26] J. Morgan and G. Tian, Ricci flow and the Poincaré conjecture, Clay Mathematics Monograph, 3, American Mathematical Society, Providence, RI, 2007 [arxiv:math/0607607]; Preprint [arxiv:0809.4040]. 
[27] R. Müller, Ricci flow coupled with harmonic map heat flow, Ph.D. thesis, ETH Zurich thesis 18290, 2009, unpublished; see also Monotone volume formulas for geometric flows, J. Reine Angew. Math. (Crelle), to appear [arxiv:0905.2328].

[28] T.A. Oliynyk, An existence proof for the gravitating BPS monopole, Ann. Henri Poincaré 7 (2006), 199-232.

[29] T.A. Oliynyk and E. Woolgar, Rotationally symmetric Ricci flow on asymptotically flat manifolds, Comm. Anal. Geom. 15 (2007), 535-568 [arXiv:math/0607438].

[30] G. Perelman, The entropy formula for the Ricci flow and its geometric applications, Preprint [arxiv:math/0211159].

[31] G. Perelman, Ricci flow with surgery on three-manifolds, Preprint [arxiv/0303109]; B. Kleiner and J. Lott, Notes on Perelman's papers, Preprint [arxiv:math/0605667].

[32] P. Petersen, Riemannian geometry, $2^{\text {nd }}$ ed., Graduate Texts in Mathematics, 171, Springer, Berlin, 2006.

[33] L.-F. Wu, A new result for the porous medium equation derived from the Ricci flow, Bull. Amer. Math. Soc. 28 (1993), 90-94; The Ricci flow on complete $\mathbb{R}^{2}$, Comm. Anal. Geom. 1 (1993), 439-472.

Dept of Mathematical and Statistical Sciences

University of AlBerta

Edmonton, AB T6G 2G1

CANADA

E-mail address: lgulcev@math.ualberta.ca

E-mail address: ewoolgar@math.ualberta.ca

School of Mathematical Sciences

Monash University

Clayton, Vic 3800

Australia

E-mail address: todd.oliynyk@sci.monash.edu.au

Received November 11, 2009 
